\title{
Tecnología web como soporte en el proceso de seguimiento del historial médico ambulatorio en el Hospital Central de la Policía Nacional del Perú, en Lima Metropolitana
}

\author{
Technology web like support in the process of pursuit of the medical ambulatory \\ record in the Central Hospital of the National Police of Peru of Metropolitan Lima
}

\author{
Silva Cachay, Jonatan*
}

http://dx.doi.org/10.21503/CienciayDesarrollo.2011.v14.07

\section{RESUMEN}

El presente trabajo de investigación tiene como objetivo el empleo de la tecnología web como soporte durante el proceso de seguimiento del historial médico ambulatorio de pacientes en el Hospital Central de la Policía Nacional del Perú, en Lima Metropolitana.

La investigación aborda el planteamiento metodológico, el marco teórico, la construcción de la herramienta y el análisis e interpretación de los resultados.

Luego de describir el planteamiento metodológico que contiene la realidad problemática, se continúa con la definición del problema y de los objetivos e hipótesis. Además, se define el tipo y nivel de investigación, así como el método de la misma.

Tras realizar la construcción de la herramienta, se exponen las características genéricas de la aplicación informática, el análisis del sistema y el diseño del mismo.

Finalmente, se hace la prueba empírica para la recopilación, análisis e interpretación de los resultados obtenidos, en el marco de la prueba de la hipótesis.

Palabras clave: Tecnología web, fiabilidad, eficiencia, base de datos.

\section{ABSTRACT}

The present investigation work has as objective the employment of the technology web like support during the process of pursuit of the medical ambulatory record of patient in the Central Hospital of the National Police of Peru, in Metropolitan Lima.

The investigation approaches the methodological position, the theoretical mark, the construction of the tool and the analysis and interpretation of the results.

After describing the methodological position that contains the problematic reality, you continues with the definition of the problem and of the objectives and hypothesis. Also, is defined the type and investigation level, as well as the method of the same one.

After carrying out the construction of the tool, the generic characteristics of the computer application, the analysis of the system and the design of the same one are exposed.

Finally, the empiric test is made for the summary, analysis and interpretation of the obtained results, in the mark of the test of the hypothesis.

Key words: Technology web, reliability, efficiency, database.

\footnotetext{
* Ingeniero de sistemas e informática. El presente trabajo es la versión resumida y adaptada de una investigación
} que el autor presentó como tesis para optar el título de Ingeniero de Sistemas e Informática en la UAP. 


\section{INTRODUCCIÓN}

Los centros hospitalarios están en la necesidad de proveer a sus pacientes un servicio rápido y eficiente, de tal manera que el paciente se sienta satisfecho con el servicio prestado por el hospital. Una de las maneras de conseguir esto es brindándole al paciente servicios con los cuales la competencia no cuente, y para lograr eso es necesario contar con un proceso de seguimiento del historial médico ambulatorio del paciente que se interrelacione con los distintos hospitales de la organización mediante un soporte tecnológico, en este caso el uso de una intranet.

En este contexto, resulta evidente la deficiencia en el control que se aplica al seguimiento del historial médico ambulatorio del paciente, ya que este proceso se hace de una manera no automatizada, lo que lo hace muy lento, engorroso y complejo, con lo que se produce un mayor índice de riesgo de que se ingrese un valor erróneo así como el riesgo de mermas en el registro de nuevos historiales médicos ambulatorios.

Actualmente la Policía Nacional del Perú cuenta con 3 hospitales y 26 policlínicos en Lima trabajando aisladamente. Esto significa que las actividades realizadas en un hospital no son comunicadas a los demás hospitales, con la consecuente incomodidad para los pacientes al momento de realizarse una consulta en un hospital distinto al que normalmente acude, puesto que para una adecuada atención se hace necesario contar con el historial médico del paciente, el mismo que se encuentra en otro hospital.

Finalmente debido a lo complejo que resulta tener la información disponible y de manera actualizada en el hospital de la Policía Nacional del Perú, la gestión del proceso de seguimiento del historial médico ambulatorio del paciente se ve afectada desde el momento de registrar el historial hasta la obtención de dicho historial en algún hospital de la organización, por la demora y los posibles errores que se suelen presentar, como la pérdida de documentos y la desactualización de los mismos. El resultado es el permanente reclamo de los pacientes y la consecuente mala imagen de la organización.

\section{Formulación del problema}

¿De qué manera la tecnología web aplicada al proceso de seguimiento del historial médico ambulatorio de pacientes influye en la gestión administrativa en el hospital central de la PNP de Lima Metropolitana?

\section{Objetivo de la investigación}

Determinar la manera en que la tecnología web aplicada al proceso de seguimiento del historial médico ambulatorio de pacientes influye en la gestión administrativa en el Hospital Central de la PNP de Lima Metropolitana

\section{Hipótesis de la investigación}

Si se aplica la tecnología web al proceso de seguimiento del historial médico ambulatorio de pacientes, se mejora la gestión administrativa en el Hospital Central de la PNP de Lima Metropolitana

\section{Variables e indicadores}

\section{Variable independiente}

$\mathrm{X} 1=$ Tecnología web como soporte al proceso de seguimiento del historial médico ambulatorio de pacientes.

\section{Indicadores}

$\mathrm{X}_{11}=\%$ Integridad de datos.

$\mathrm{X}_{12}=\%$ Fiabilidad. 


\section{Tabla 1. Indicadores e índices (variable independiente)}

\begin{tabular}{|c|c|}
\hline INDICADORES & ÍNDICES \\
\hline $\mathrm{X}_{11}=\%$ Integridad de datos & $\begin{array}{l}\text { Cantidad de historiales médicos ambulatorios con información } \\
\text { actualizada sin errores con tecnología web al día/ Cantidad de } \\
\text { historiales médicos ambulatorios con información actualizada } \\
\text { sin errores sin tecnología web al día. }\end{array}$ \\
\hline$X_{12}=\%$ Fiabilidad & $\begin{array}{l}\text { Cantidad de respuestas sin errores a consultas de historiales } \\
\text { médicos ambulatorios con tecnología web por día / Cantidad } \\
\text { de respuesta sin errores a consultas de historiales médicos } \\
\text { ambulatorios sin tecnología web por día. }\end{array}$ \\
\hline
\end{tabular}

\section{Variable dependiente}

Y1= Influencia en la gestión administrativa en el Hospital Central de la PNP de Lima Metropolitana.

\section{Indicadores}

$\mathrm{Y}_{11}=\%$ Eficiencia.

Tabla 2. Indicadores e índices (variable dependiente)

\section{INDICADORES}

$\mathrm{Y}_{11}=\%$ Eficiencia

\section{ÍNDICES}

Tiempo promedio en buscar un historial médico sin tecnología Web por día / Tiempo promedio en buscar un historial médico con tecnología web por día.

\section{MATERIAL Y MÉTODO}

Para el desarrollo de esta investigación se ha utilizado el método científico debido a que proporciona un planteamiento ordenado y un alto nivel de rigurosidad con respecto al tratamiento de los datos y el análisis de los resultados. También se ha utilizado el concepto sistémico, ya que nos permite tener una visión y un entendimiento integral de toda la situación problemática en estudio, así como nos brinda un mejor enfoque de la solución.

Sobre la base de lo explicado anteriormente, el presente trabajo de investigación sigue un método comprobado de recopilación, tabulación y análisis de los antecedentes que se han obtenido y comprobado directamente en el campo donde se ha realizado el trabajo de investigación.

\section{Diseño de la investigación}

El diseño seleccionado para el desarrollo de la investigación fue el experimental porque proporciona al investigador la seguridad de que los resultados observados responden a la variable experimental o independiente utilizada. Concretamente se trata de un diseño de prueba. Este diseño incluye dos grupos, uno que recibe el tratamiento experimental y otro que no, es decir, el tratamiento de la variable independiente alcanza solo dos niveles (presencia y ausencia). 
El diseño puede representarse utilizando el siguiente diagrama:

$$
\begin{array}{ccc}
\text { G1 } & \text { X } & \text { P1 } \\
\text { G2 } & - & \text { P2 }
\end{array}
$$

Se trata de una conformación aleatoria de un grupo (G1) formado por la cantidad de historiales médicos ambulatorios de pacientes, al que se le administra un tratamiento experimental. La tecnología web como soporte al proceso de seguimiento del historial médico ambulatorio de pacientes viene definida por $\mathrm{X}$. A este tratamiento se le aplica una prueba posterior definida por P1. Al segundo grupo (G2), conformado también por la cantidad de historiales médicos ambulatorios de pacientes, no se le administra ningún tratamiento experimental, sirviendo solamente como grupo de control. A este grupo en forma simultánea se le aplica la prueba P2.

Los dos grupos están formados de manera aleatoria pero son estadísticamente representativos, por las operaciones realizadas tanto en ausencia como en presencia de la tecnología de la información propuesta.

\section{Técnicas e instrumentos de recolección de información}

Las técnicas e instrumentos utilizados para la recopilación procesamiento y despliegue de la información corresponden a los que se emplean generalmente en este tipo de investigaciones.

\section{Técnicas}

Las técnicas que se han utilizado para el levantamiento de la información son:

- Encuestas (Anexo 1).

- Entrevista (Anexo 2).

- Análisis documental.

- Cronometraje de tiempo de proceso (observación de campo) (Anexo 3).

\section{Instrumentos}

Los instrumentos utilizados fueron los siguientes:

- Cuestionario (Anexo 1).

- Guía de entrevista (Anexo 2).

- Fichas Técnicas.

- Tabla de tiempos de observación del proceso (Anexo 3).

\section{Cobertura del estudio}

\section{Universo}

La unidad de análisis que sirve como base para la definición de la población está conformada por las atenciones de los pacientes ambulatorios efectuadas en el marco del proceso de seguimiento del historial médico ambulatorio de pacientes en el Hospital Central de la PNP de Lima Metropolitana.

\section{Muestra}

La muestra viene dada por el número representativo de atenciones de los pacientes ambulatorios en el marco del proceso de seguimiento del historial médico ambulatorio de pacientes en el Hospital Central de la PNP de Lima Metropolitana.

\section{Tecnología web}

La web creó un mercado casi perfecto, eliminando mucho de los costos de tiempo $y$ distancia. La web posibilita que las organizaciones alcancen a las personas que afectan a sus operaciones, inclusive proveedores, socios y unidades de negocio (o departamentos) de la empresa. Los clientes y proveedores pueden acceder a las informaciones en vez de usar intermediarios. 
Los sistemas de flujo de trabajo automatizan los procedimientos por los cuales los documentos, las informaciones y las tareas son distribuidos dentro de la empresa. Estos sistemas aplican reglas de trabajo definidas en secuencias de actividades. El flujo de trabajo ofrece un modelo de procesos de negocios centrados en el cliente, como el gerenciamiento de pedidos y la atención al cliente, y permite también a los usuarios direccionar las comunicaciones con los clientes junto con una carpeta virtual asociada que combina documentos, mensajes de voz, e-mail, faxes, videos o páginas web que ofrecen informaciones sobre los clientes. Estas informaciones pueden ser transmitidas a los puntos de atención al cliente adecuados en el momento apropiado para ofrecer una solución a los clientes usando cualquier canal.

\section{Requerimientos de hardware}

- Un servidor donde se centralizará la información, y en el que se almacenarán la base de datos de los historiales médicos, el sistema web y cualquier aplicativo necesario.

- Dos computadoras con las configuraciones necesarias para el desarrollo del aplicativo web.

- Router, switch y el cableado necesario para la conexión interna de la intranet y acceso a internet para la empresa, además del cableado para la instalación y uso de internet y redes tanto en los equipos de trabajo como en el servidor.

\section{Tabla 3. Requerimientos técnicos del hardware}

\section{REQUERIMIENTOS $\quad$ CANTIDAD}

\begin{tabular}{ll} 
Servidor de Base de datos & 1 \\
$\begin{array}{l}\text { Estaciones de trabajo } \\
\text { (Computadoras) }\end{array}$ & 2 \\
Router & 1 \\
Switch & 1 \\
Cable UTP & 2 \\
\hline
\end{tabular}

\section{Requerimientos de software}

- Un software gestor de base de datos que pueda interactuar con el aplicativo web.

- El sistema operativo servidor, el cual va a soportar toda la aplicación y las transacciones del sistema.

- Un software para el desarrollo de aplicaciones web orientadas a objetos.

- Una herramienta case que dé soporte a cada una de las fases del proceso unificado de desarrollo.

- Internet, para mantener una línea de acceso interna y externa que permita la realización de pruebas del aplicativo y la comunicación entre el hospital central y las filiales.

- Un antivirus corporativo que permitirá tener un nivel de seguridad adecuado.

- Un software de asistencia remota.

\section{Tabla 4. Requerimientos técnicos del software}

\section{REQUERIMIENTOS}

\section{SISTEMAS OPERATIVOS SERVIDOR}

Microsoft Windows Server 2003

Windows XP Profesional

BASE DE DATOS

SQL Server 2005

SOFTWARE

Microsoft Visual Studio.Net 2005

Rational Rose 2003

ESET Nod32 Antivirus 
Requerimientos para el desarrollo del sistema

Tabla 5. Requerimientos para el desarrollo del sistema

\begin{tabular}{l|l|l}
\multirow{2}{*}{\multicolumn{1}{c}{ EQUIPO }} & \multicolumn{2}{c}{ DESCRIPCION } \\
\cline { 2 - 3 } & \multicolumn{1}{c}{ DESARROLLO } & \multicolumn{1}{c}{ SERVICIOS } \\
\hline PROCESADOR & Core 2 Duo & Core Quad \\
\hline VELOCIDAD & $2.4 \mathrm{Ghz}$ & $2.8 \mathrm{GHZ}$ \\
\hline HP & $160 \mathrm{~Gb}$ & $500 \mathrm{~Gb}$ SCSI \\
RAM & $2 \mathrm{~Gb}$ & $8 \mathrm{~Gb}$ \\
\hline TECLADO & Doble golpe 101 & Doble golpe 101 \\
MOUSE & Sin especificación & Sin especificación \\
\hline MONITOR & Samsung NE 17" & Samsung NE 17" \\
TARJETA DE RED & Integrada & Integrada \\
\hline
\end{tabular}

Cables de red, puntos de conexión, tomas de corriente eléctricas, supresores, switch, router, etc.

\begin{tabular}{c} 
LICENCIAS \\
Software \\
Microsoft SQL Server 2005 \\
Windows 2003 Server \\
Windows XP \\
Microsoft Visual Studio 2005 \\
\hline
\end{tabular}

\section{Modelo de negocio}

El modelo de negocio es una técnica cuyo objetivo es conocer cómo funcionan los procesos de negocio de la empresa.
En la figura 1 se detalla el macroproceso y sus respectivos procesos.

En los siguientes gráficos se observa cómo funciona el negocio dentro del proceso de seguimien-

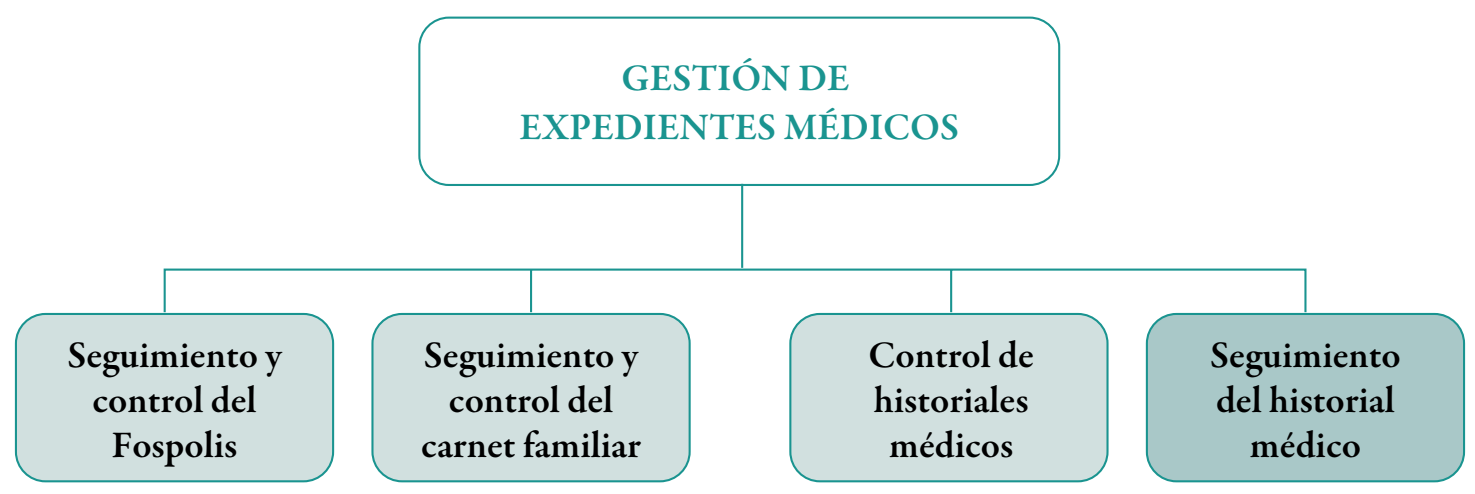

Fuente: el autor.

Figura 1. Proceso de seguimiento al historial médico ambulatorio de pacientes 
to del historial médico ambulatorio de pacientes en el Hospital de la Policía Nacional del Perú.

Este proceso comienza cuando el paciente solicita realizar su trámite de creación de su historial médico. Posteriormente, el encargado del registro de dicho historial procederá a crear su archivo médico. Luego de la creación satisfactoria del historial médico, este se deriva al almacén, donde dicho historial será guardado y puesto a disposición del encargado del transporte de documentos para posteriormente ser enviado al consultorio correspondiente, dependiendo de la cita que se le haya otorgado al paciente.

El transporte del historial médico se realiza en los horarios establecidos en los consultorios asignados por la cita otorgada al paciente de dicho historial.

Después de conocer el flujo del proceso de seguimiento del historial médico ambulatorio, surge la necesidad de saber cómo este proceso influye en la gestión administrativa del hospital de la Policía Nacional del Perú. Entonces, partiendo de la premisa de que una empresa es un sistema, y que este está conformado por procesos, y este a su vez por actividades, concluimos que la gestión administrativa es un proceso que se encarga de la planificación, organización, ejecución y control dirigidos a alcanzar los objetivos propuestos. Además, sabemos que por TGS, una de las características de los sistemas es el globalismo o totalidad, característica que explica que si existe

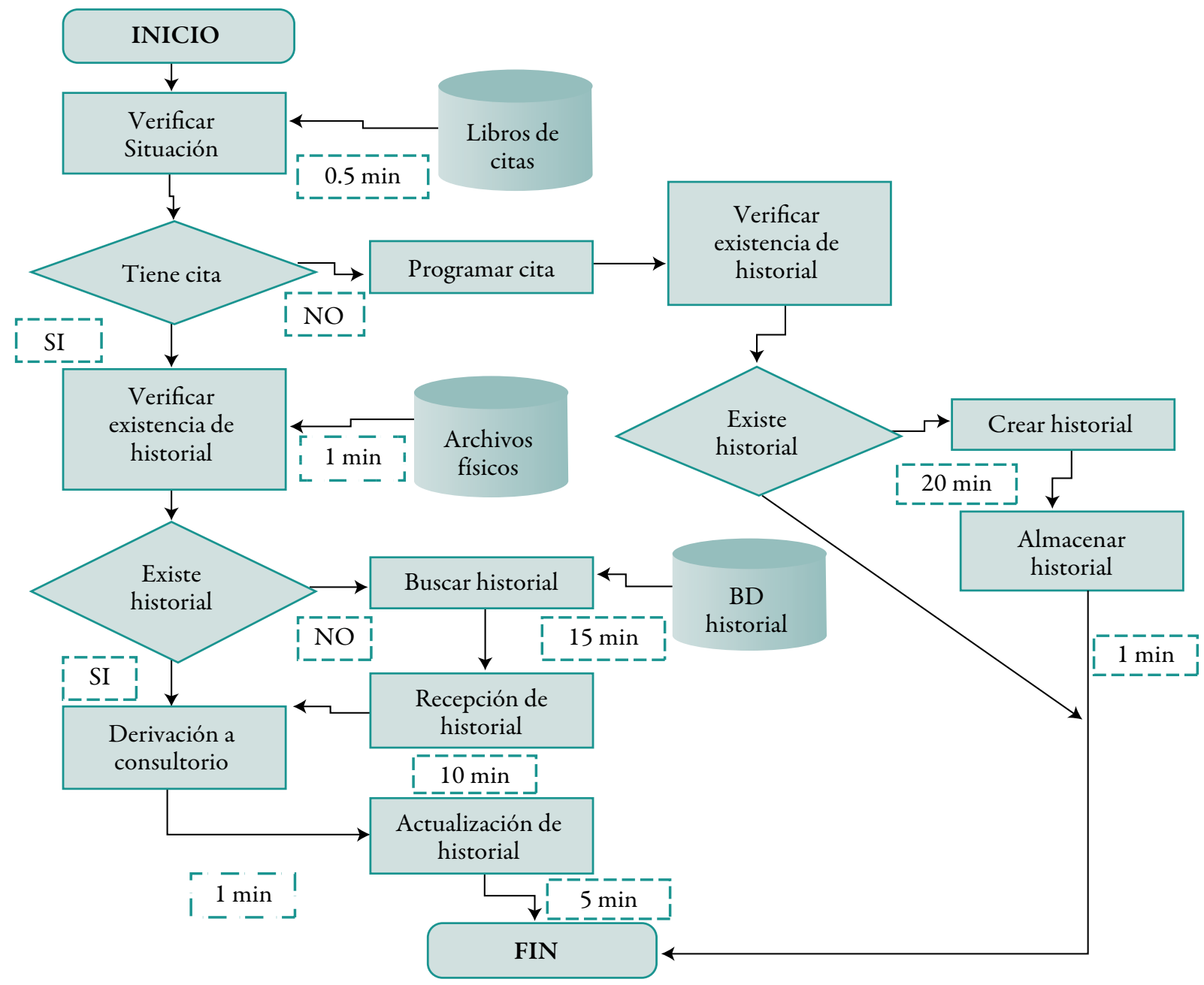

Fuente: el autor.

Figura 2. Proceso de seguimiento al historial médico ambulatorio de pacientes 
algún cambio en una de las unidades del sistema, probablemente se producirán cambios en las otras.

Así, según lo anterior, sabemos que si algún proceso del sistema funciona mal, ello redundará negativamente en los demás procesos, ya que si hablamos de sistema estamos hablando de un todo, de modo que al influir negativamente en los demás procesos provocará un efecto negativo en la gestión administrativa, puesto que va a impedir que se cumplan los objetivos ya planificados.

En la figura 2 se observa el modelo de negocio del proceso de seguimiento del historial médico ambulatorio de pacientes, los actores involucrados en este proceso y cómo interactúan unos con otros, desde la gestión de la información del paciente para la creación del historial médico hasta su actualización.

Según la figura 2, el proceso de seguimiento del historial médico ambulatorio de pacientes, el modo en que interactúan las actividades y el almacén de datos (almacén físico), se generan numerosos problemas con respecto a la información, que no resulta por ello confiable. Además, el tiempo perdido en la búsqueda y la información no están en tiempo real.

\section{Diseño de la base de datos}

\section{Modelo de datos}

Un modelo de datos se construye y representa gráficamente por el diagrama de modelo de datos. El modelo de datos se usa para capturar

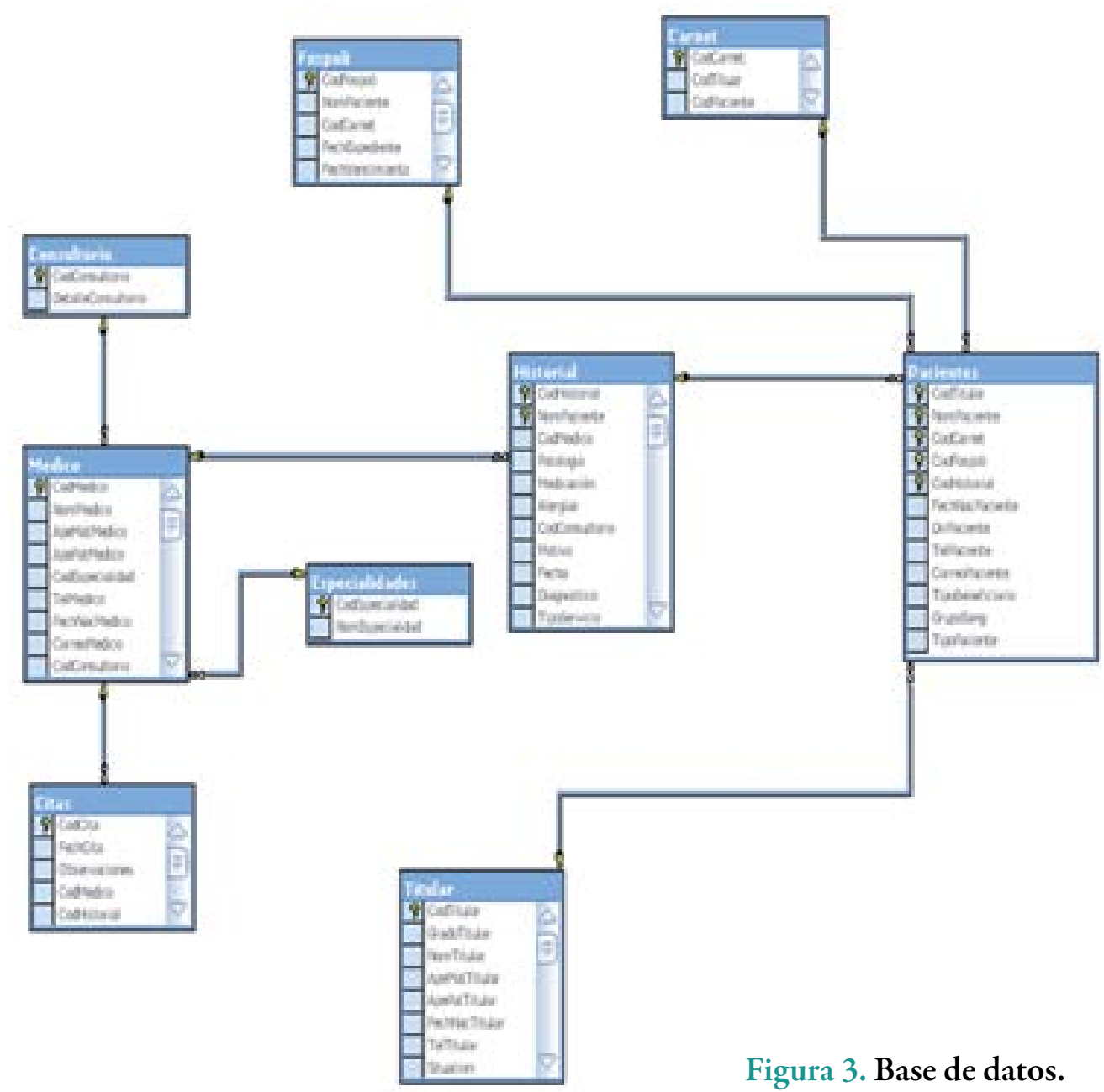


una vista física de la base de datos. Los modelos de datos son dependientes de la base de datos. El modelo de datos es diseñado para crear un esquema en la vista lógica y asignarlo a una base de datos en la vista del componente. Luego se asignan las tablas al esquema, se asignan las columnas a las tablas y los key constraints a las columnas para asegurar la integridad referencial del modelo. Además, se puede agregar triggers (disparadores), check constraints, índices, y stored procedures (procedimientos almacenados) para adicionar funcionalidad.

Este diagrama de base de datos representa los objetos que pertenecen a la base de datos como entidades que tienen unos atributos y se vinculan mediante relaciones.

\section{Diseño de la interfaz grafica de usuario}

A continuación se mostrarán y describirán las principales pantallas que la aplicación emplea para la administración del sistema.

\section{Interfaz gráfica de login}

El sistema como medio de seguridad le pedirá un usuario y contraseña para poder ingresar al sistema, y además validará el tipo de usuario y sus respectivos privilegios.

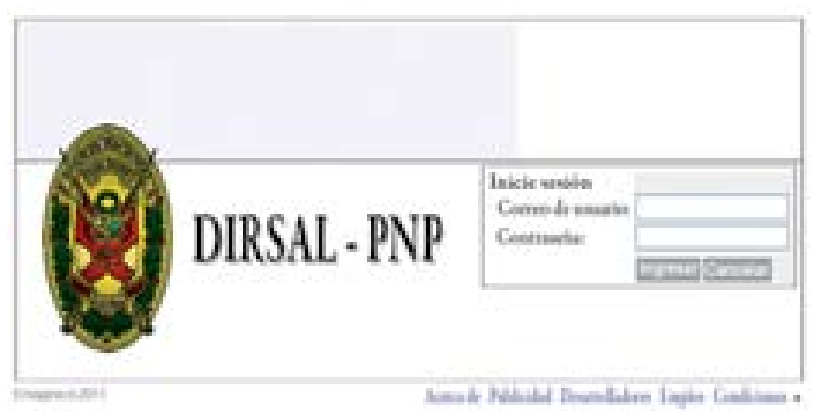

Figura 4. Interfaz de login

\section{Información básica del sistema}

En la siguiente pantalla se presenta el módulo del doctor, en donde se podrá ingresar rápidamente a buscar las citas de algún paciente, las citas del día, un historial médico, así como registrar un nuevo historial médico.

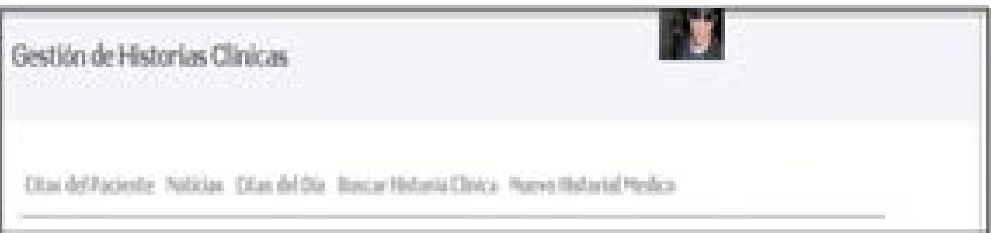

\begin{tabular}{l|l}
\hline Citas del día & $\begin{array}{l}\text { Nos proporciona una pantalla de búsqueda del paciente para la } \\
\text { pronta actualización de su historial. }\end{array}$ \\
\hline Buscar historia clínica & $\begin{array}{l}\text { En este módulo se muestra una pantalla para dar mantenimiento a los } \\
\text { pacientes (Eliminar, Nuevo Actualizar). }\end{array}$ \\
\hline Citas del paciente & $\begin{array}{l}\text { Las citas se dividen en dos pantallas. La primera es donde se podrá } \\
\text { hacer una búsqueda o listar las citas según algún criterio de búsqueda } \\
\text { (Citas del día, Citas X, Consultorio, etc.), y la otra pantalla es de } \\
\text { detalle para dar el respectivo mantenimiento a las citas (Nueva, } \\
\text { Actualizar, Eliminar). }\end{array}$ \\
\hline Nuevo historial médico & Modulo de creación de nuevo historial médico. \\
\hline Mantenimiento del historial & Todas las búsquedas llevan a un mantenimiento de historial médico. \\
\hline
\end{tabular}

Figura 5. Información básica 


\section{Interfaz menú principal}

Esta interfaz es la pantalla principal que contiene todos los módulos que conforman el sistema de gestión de documentos (historias médicas) de la Policía Nacional del Perú.
Este módulo, denominado módulo del doctor, cuenta con un sistema de seguridad que permite gestionar usuarios del sistema y roles de los usuarios. Este modulo cuenta con información del paciente, y además permite hacer un seguimiento a los tratamientos y citas del paciente.

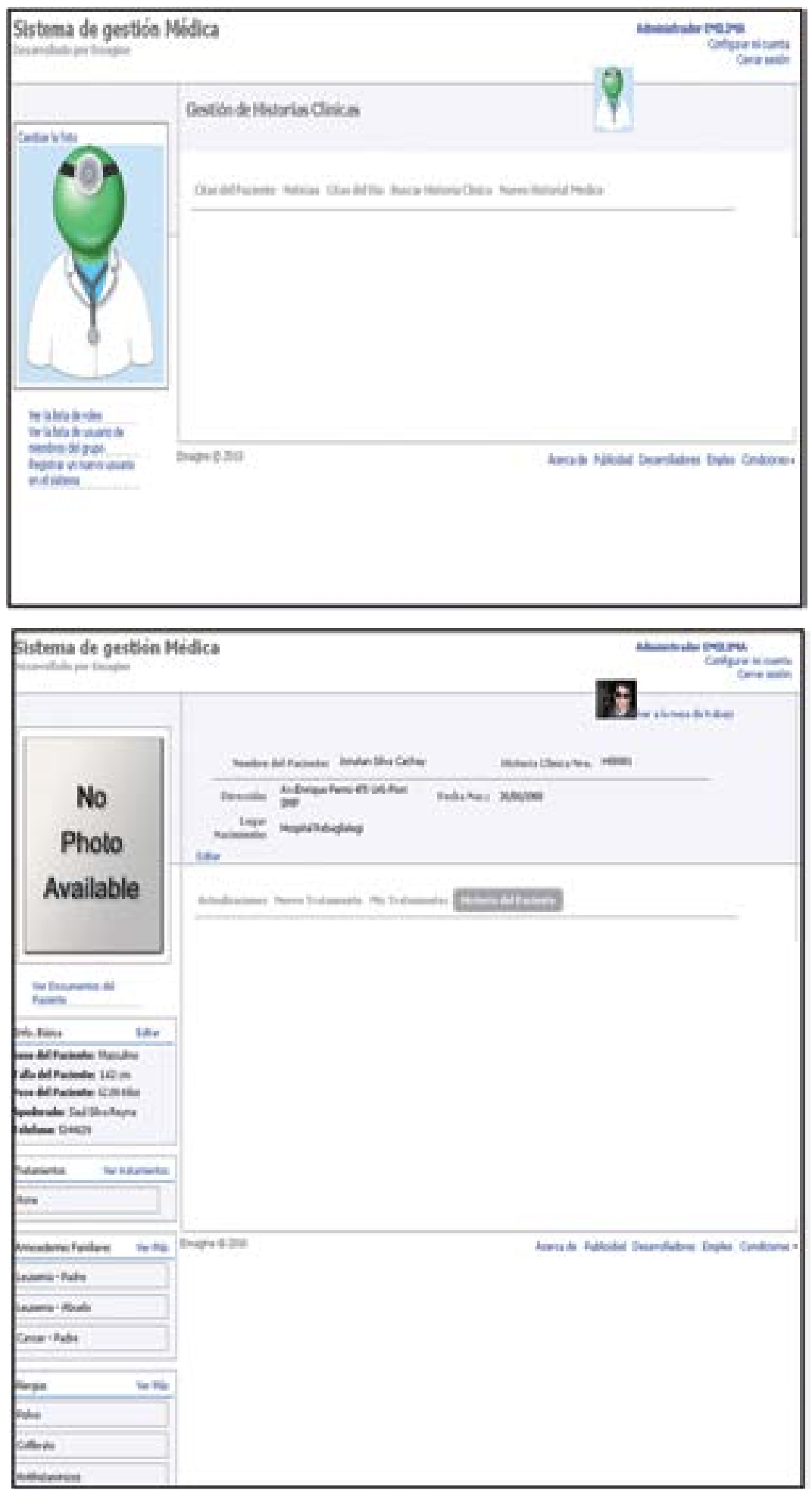

Figura 6. Menú principal 


\section{Interfaz consulta del día/búsqueda}

Esta interfaz nos proporciona una pantalla de búsqueda del paciente para la pronta actualización de su historial.

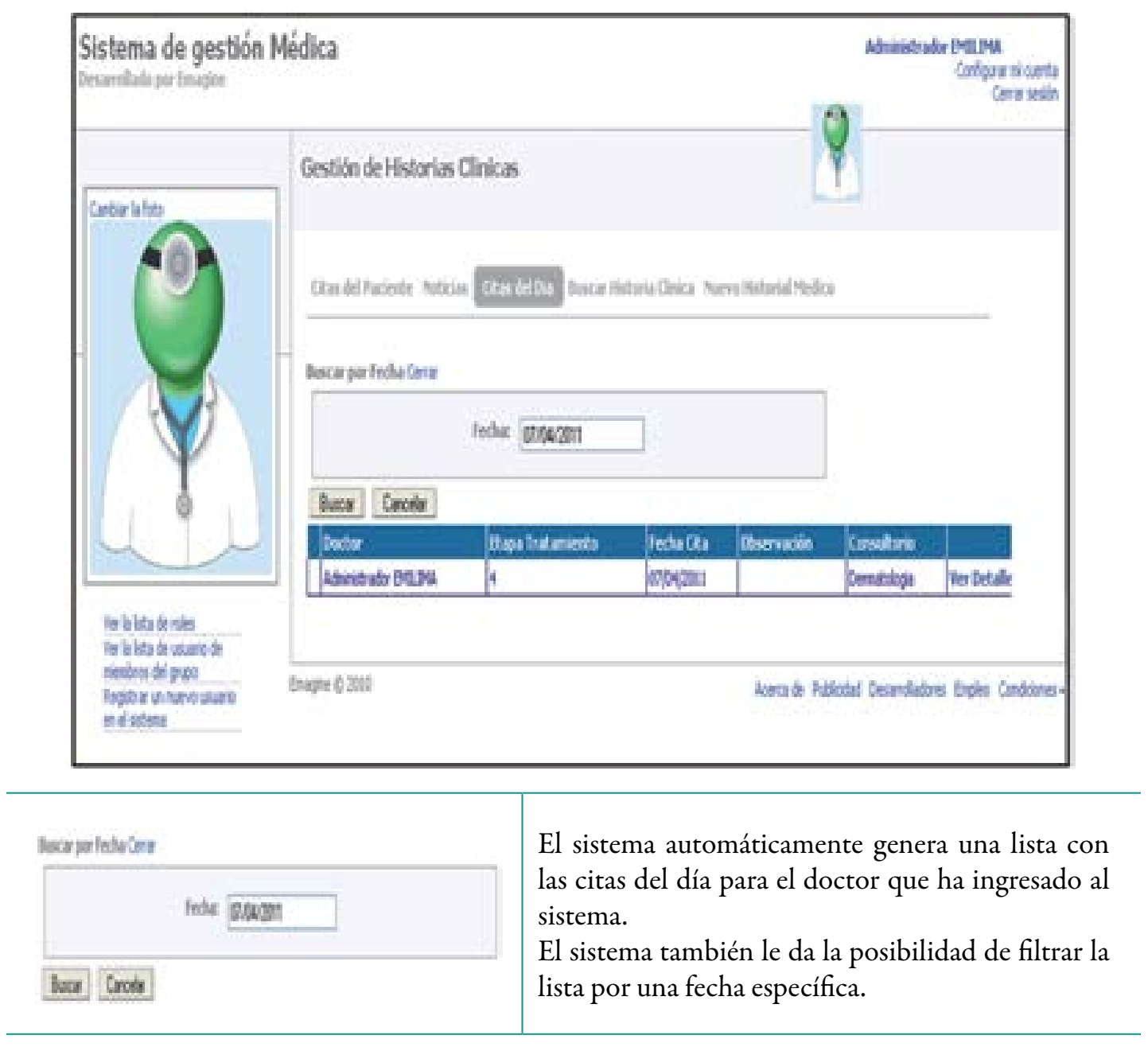

Figura 7. Consulta del día/búsqueda 


\section{Interfaz consulta del día/búsqueda: segunda pantalla}

Esta interfaz sirve para dar el respectivo mantenimiento al historial del paciente.

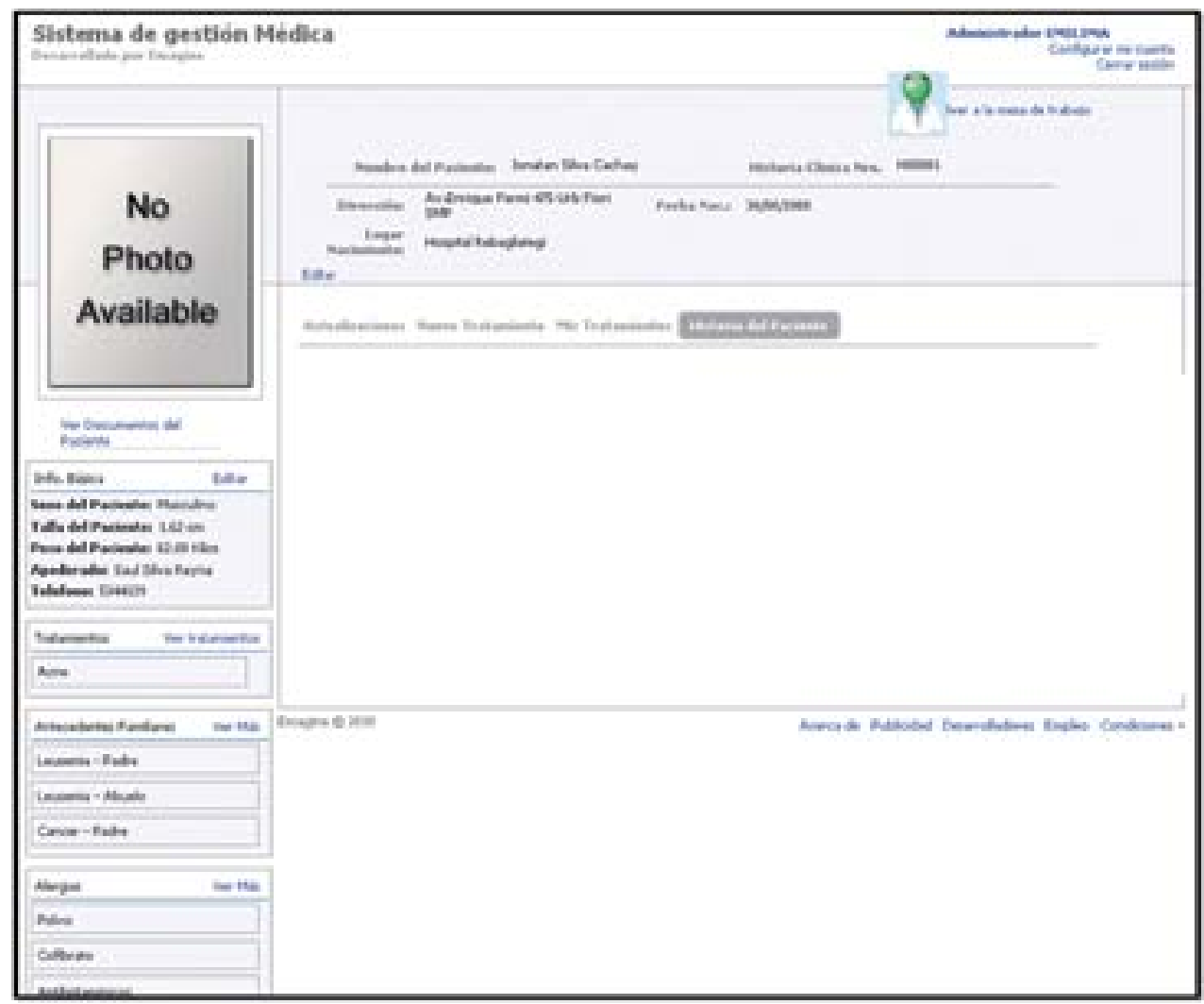

\begin{tabular}{|c|c|}
\hline Alergat & \multirow[t]{4}{*}{$\begin{array}{l}\text { En la casilla Alergias, el médico puede agregar nuevas alergias } \\
\text { encontradas al paciente, para que otros médicos las tengan en } \\
\text { cuenta al recetar algún medicamento. }\end{array}$} \\
\hline Colteroto & \\
\hline Anthisenminicos & \\
\hline Conotedns & \\
\hline Antecedertes Fonitres & \multirow{4}{*}{$\begin{array}{l}\text { En la casilla Antecedentes Familiares, se hace referencia a } \\
\text { enfermedades que han sufrido familiares del paciente. } \\
\text { El médico puede agregar nuevos antecedentes familiares para que } \\
\text { otros médicos los tengan en cuenta al recetar algún medicamento. }\end{array}$} \\
\hline Lensiemia = Podro & \\
\hline Leusemis - Abueto & \\
\hline Concer - Pobse & \\
\hline Ver bretensientos & \multirow{2}{*}{$\begin{array}{l}\text { La casilla Tratamientos se actualiza con los últimos } 6 \text { tratamientos } \\
\text { del paciente, para tener un historial de tratamientos. }\end{array}$} \\
\hline Aene & \\
\hline Wha etaies Eder & \multirow{2}{*}{$\begin{array}{l}\text { En la casilla Información Básica se muestra información relevante } \\
\text { del paciente. }\end{array}$} \\
\hline 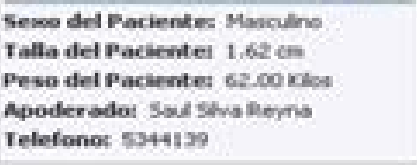 & \\
\hline 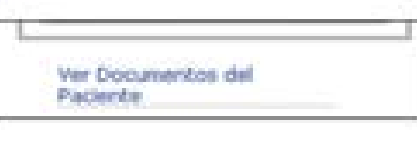 & $\begin{array}{l}\text { Cada paciente tiene un módulo de documentos adjuntos en } \\
\text { donde se almacenarán documentos como rayos } \mathrm{X} \text { o algún otro } \\
\text { documento que el doctor considere relevante. }\end{array}$ \\
\hline
\end{tabular}

Figura 8. consulta del día/búsqueda: segunda pantalla 


\section{Interfaz nuevo historial médico}

Esta interfaz permitirá ingresar los datos necesarios para la creación de nuevo historial médico.

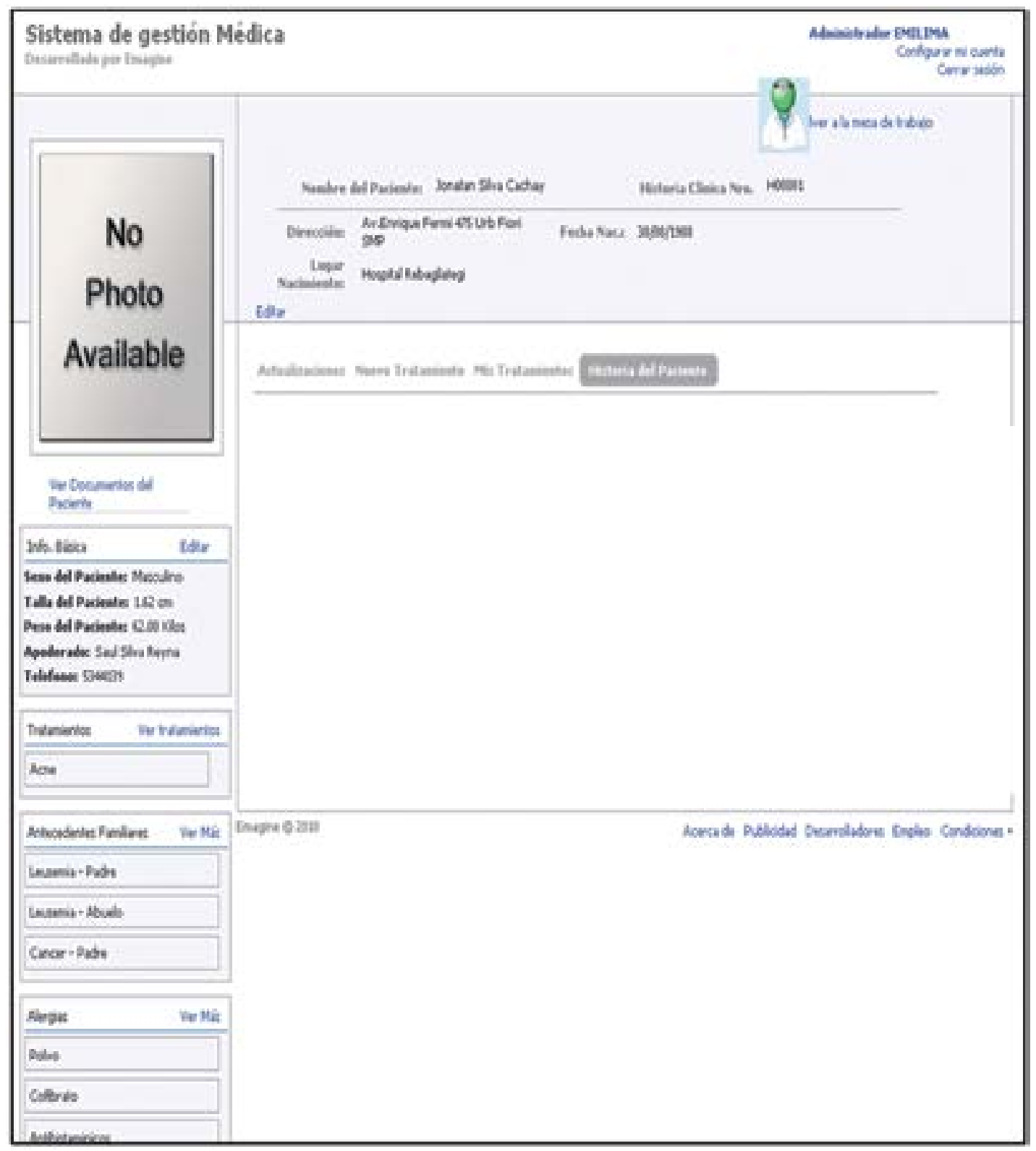

Figura 9. Nuevo historial médico 


\section{Interfaz mantenimiento de médicos}

Esta interfaz ayuda a realizar el mantenimiento de los médicos mediante una búsqueda que permitirá acceder a una lista de médicos en la que se podrá elegir a quién dar el mantenimiento.

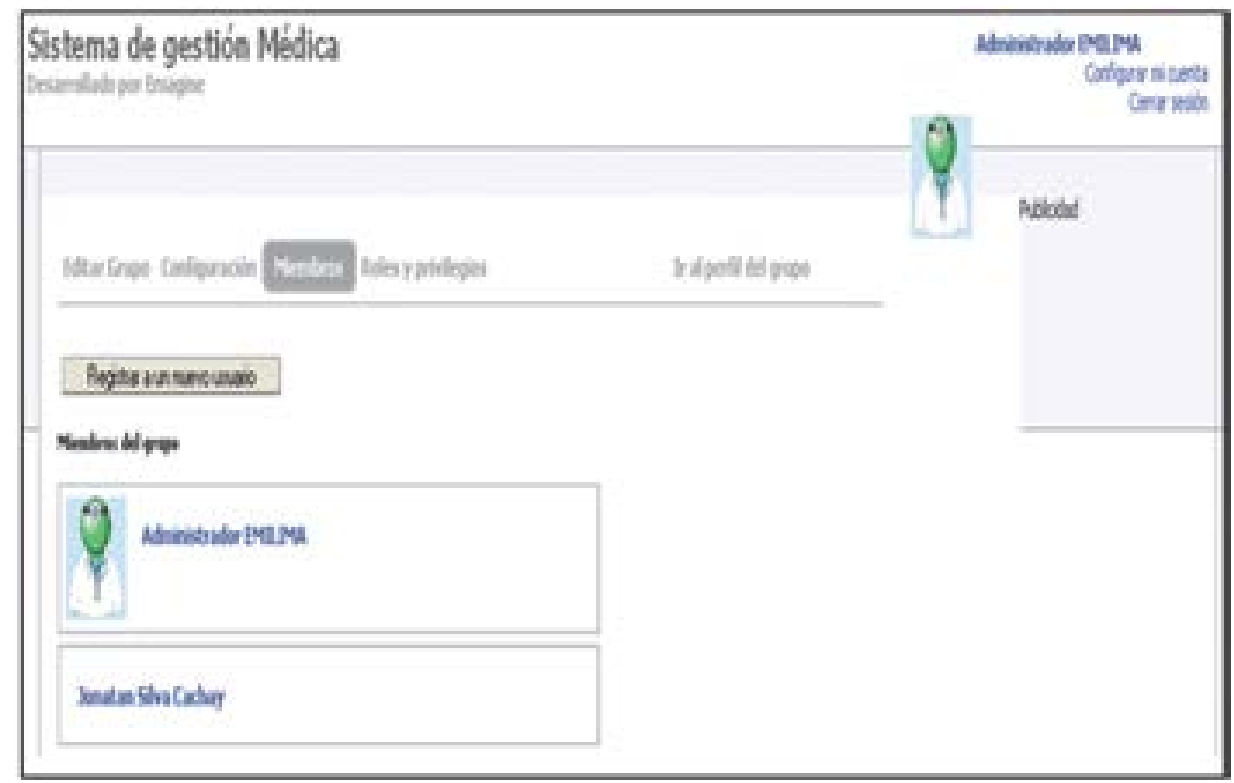

Figura 10. Mantenimiento de médicos

\section{Interfaz módulo de citas - Nueva cita}

Esta interfaz permite Ingresar los datos necesarios para la creación de una nueva cita.

Cabe mencionar que se podrá crear una cita siempre y cuando se haya tenido una consulta o tratamiento.

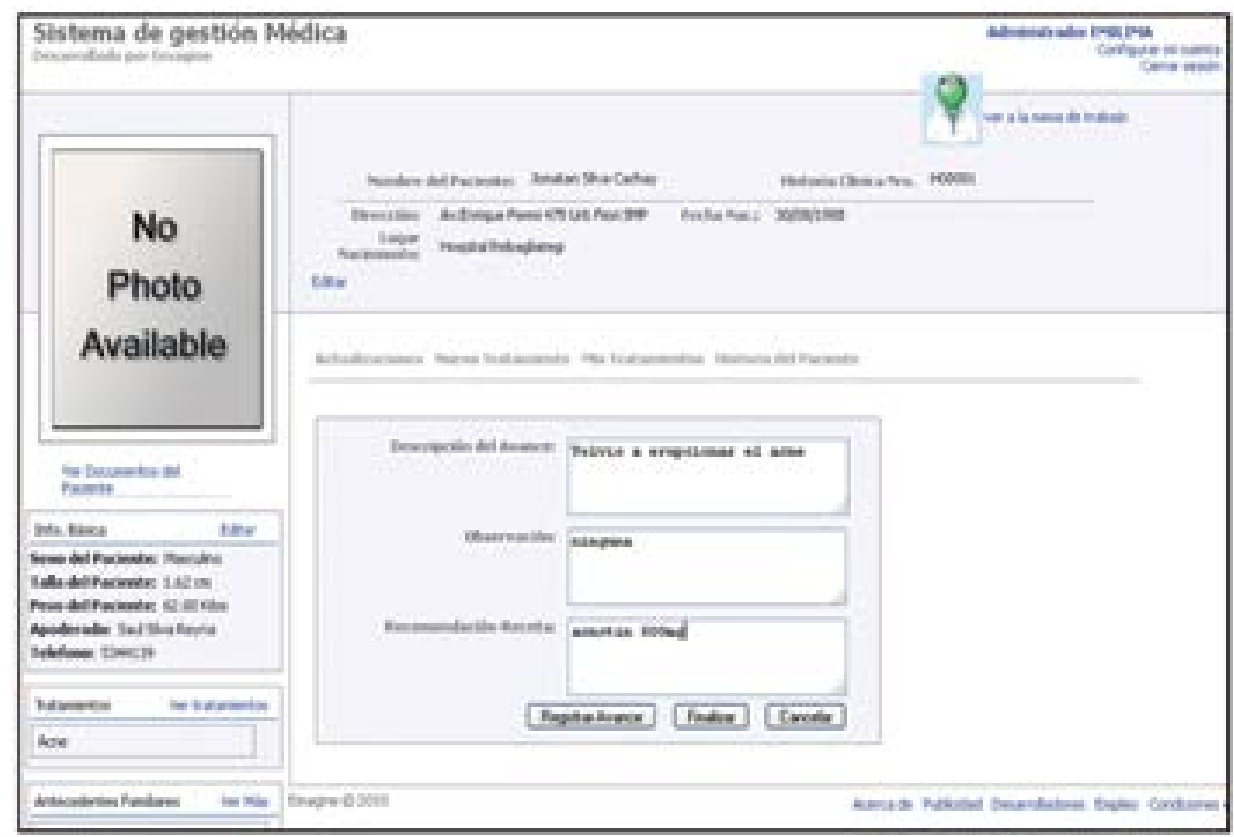

Figura 11a. Módulo de citas 


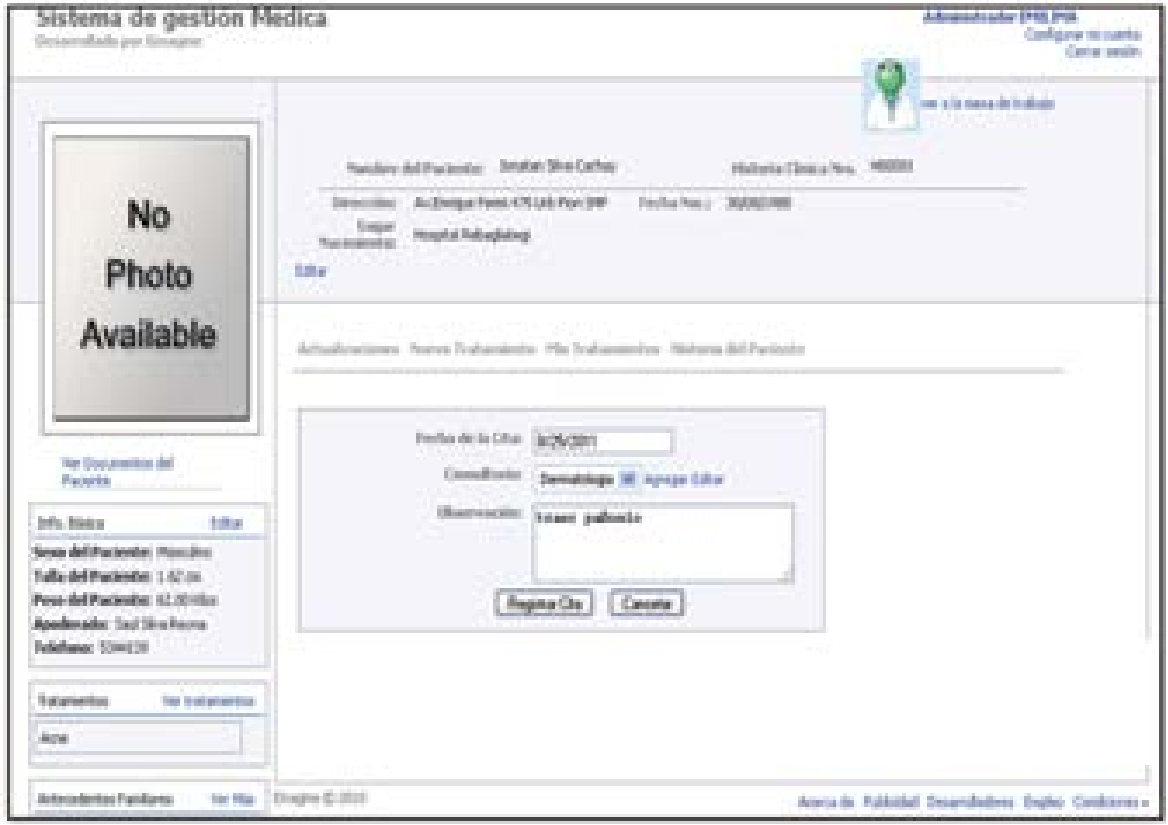

Figura 11b. Nueva cita

Interfaz módulo de citas - Mantenimiento de citas

Esta interfaz sirve para el mantenimiento de las citas mediante una búsqueda que permitirá acceder a una lista de citas en donde se podrá elegir a quién dar el mantenimiento. Además, permitirá el mantenimiento de las citas que ha tenido un paciente en un determinado tratamiento.

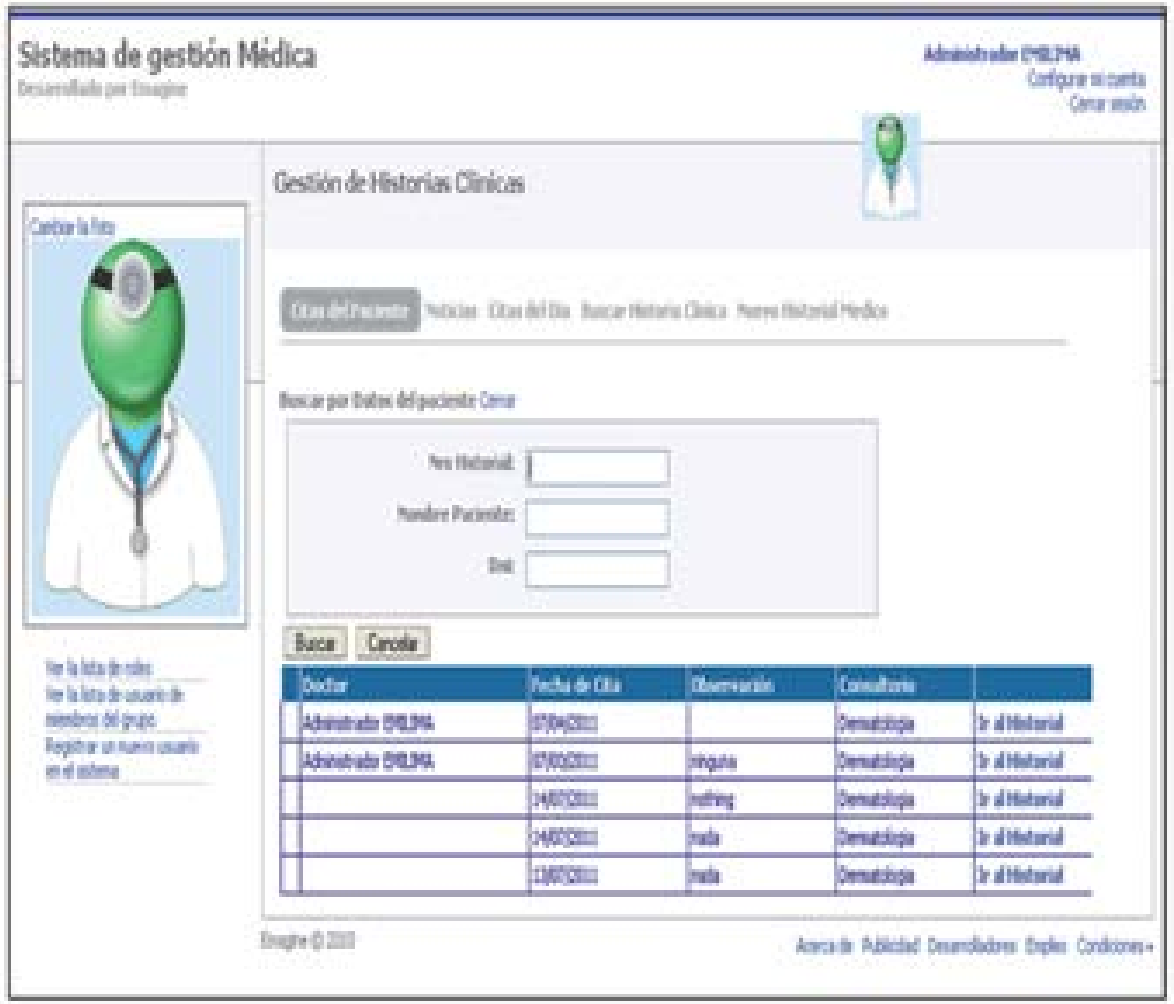

Figura 12a. Mantenimiento de citas - Cita del doctor 


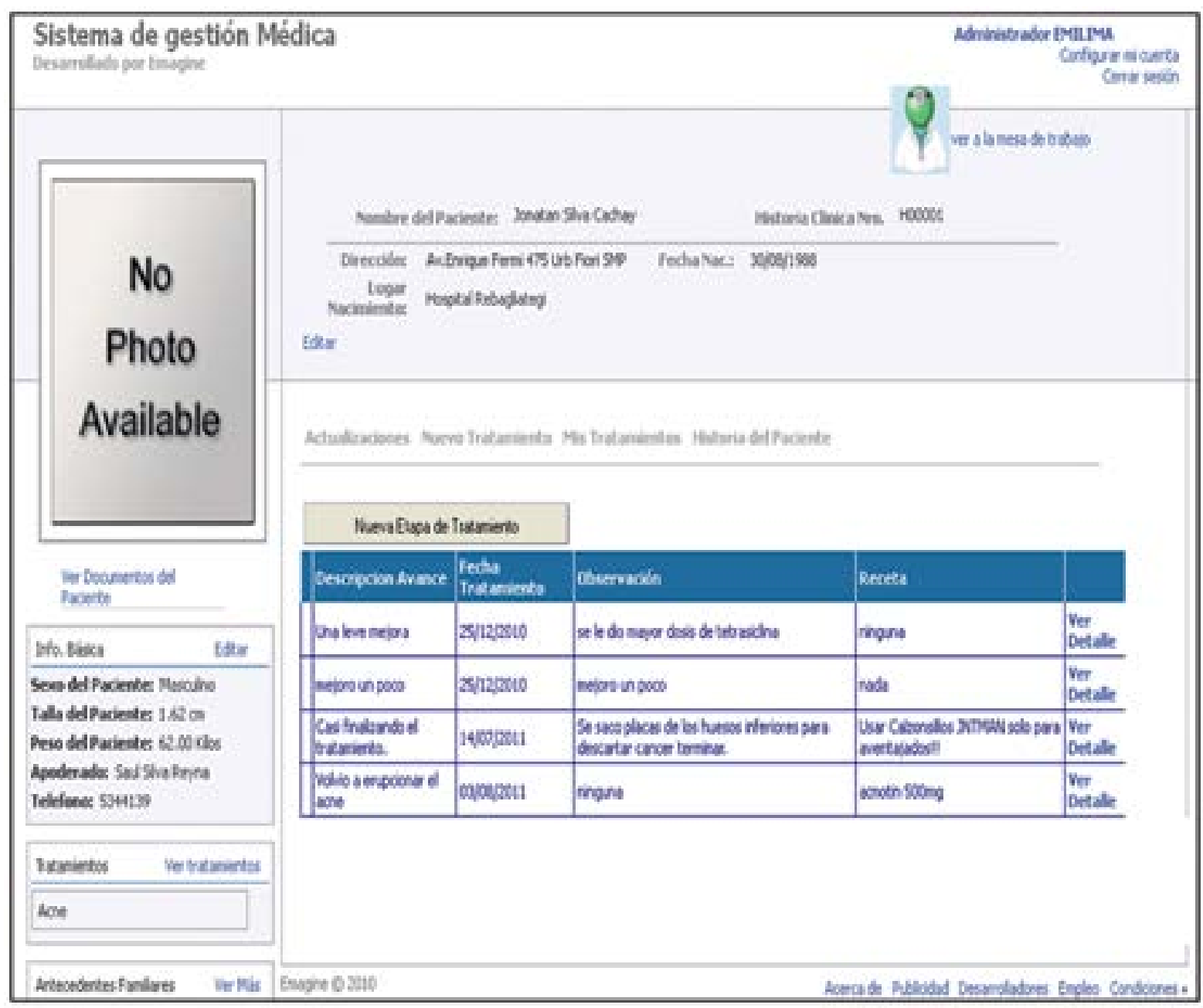

Figura 12b. Mantenimiento de citas- Citas del paciente en un determinado tratamiento

\section{ANÁLISIS E INTERPRETACIÓN DE RESULTADOS}

La ficha técnica sobre la cual van a ser probados los datos recolectados para la prueba de hipótesis está diseñada de la siguiente manera:

\section{Nivel de confianza : $\quad 95 \%$ \\ Significancia : $\quad 5 \%$}

Teniendo en consideración las características de la población, de la muestra, del nivel de confianza y de la significancia, con el propósito de que los resultados estén respaldados estadísticamente, esto es, que sean representativos, se ha seleccionado la fórmula de garantía de tamaño de muestra óptima:

\section{FÓRMULA PARA HALLAR EL TAMAÑO REPRESENTATIVO DE LA MUESTRA}

$N=\left[\frac{40 n}{\sum x} \sqrt{\frac{\sum x^{2}-\frac{\left(\sum x\right)^{2}}{n-1}}{\sum}}\right]^{2}$

Donde:

$\mathrm{N}$ : Tamaño de muestra.

$\mathrm{n}$ : Número de observaciones recopiladas por índice.

$\mathrm{x}$ : Valor de la observación.

Esta fórmula es una síntesis de garantía según tamaño de la muestra, para un nivel de aceptación del $95 \%$ y un límite de error de $5 \%$, teniendo en consideración que la unidad de análisis del 
trabajo de investigación está constituida por transacciones que tienen mucha afinidad de comportamiento con las técnicas de medición del trabajo.

A continuación se despliegan los resultados obtenidos de la prueba de campo realizada tanto para la variable independiente como para los grupos de control y experimental de la variable dependiente, aplicando las métricas correspondientes a los indicadores seleccionados. Dichos resultados son sometidos a un minucioso análisis para extraer los principales rasgos de su comportamiento y de este modo tener elementos de juicio para interpretar de manera global el comportamiento de las dos variables involucradas.

\section{Para la variable independiente}

$\mathrm{X} 1=$ Tecnología web como soporte al proceso de seguimiento del historial médico ambulatorio de pacientes.

Tabla 6. Indicadores e índices de la variable independiente

\begin{tabular}{ll} 
INDICADORES & \multicolumn{2}{c}{ ÍNDICES } \\
& Cantidad de historiales \\
& médicos ambulatorios con \\
& información actualizada sin \\
X11 $\%$ & errores con tecnología web al \\
Integridad de & día / Cantidad de historiales \\
datos & médicos ambulatorios con \\
& información actualizada sin \\
& errores sin tecnología web al \\
& día. \\
& Cantidad de respuesta sin \\
& erroresaconsultasdehistoriales \\
médicos ambulatorios con \\
tecnología web por día / \\
Cantidad de respuesta sin \\
erroresaconsultasdehistoriales \\
médicos ambulatorios sin \\
tecnología web por día.
\end{tabular}

\section{Para el indicador Integridad de datos}

En el caso de este indicador, el análisis se basa en la tabla 7.

Tabla 7. Resultados para el indicador Integridad de datos

Cantidad de historiales médicos ambulatorios con información actualizada sin errores sin tecnología web al 12,03 día.

Cantidad de historiales médicos ambulatorios con información actualizada sin 1,16 errores con tecnología web al día

Incremento de la Integridad de datos

$12,03-1,16=10,86$

\% Integridad de datos

$90,00 \%$

Según las observaciones (ver anexos 3 y 4 ), se concluyó que el tiempo promedio de respuesta a consulta de historiales médicos ambulatorios con información actualizada sin errores sin tecnología web era de 12,03 minutos. Luego de aplicar la tecnología, el tiempo promedio de respuesta fue de 1,16 minutos. Como se puede ver, el tiempo de respuesta sin errores se ha reducido en 10,86 minutos; por tanto, la fiabilidad se ha incrementado en $90,89 \%$.

\section{Para el indicador Fiabilidad}

En el caso de este indicador, el análisis se basa en la tabla 8.

Según las observaciones (ver anexos 5 y 6), se obtuvo que el tiempo promedio de respuesta sin errores a consultas de historiales médicos ambulatorios sin tecnología web por día era de 8,53 minutos. Luego de aplicar la tecnología, el tiempo promedio de respuesta resultó de 1,63 minutos. Como se puede ver, el tiempo de 
respuesta sin errores se redujo en 6,9 minutos; por tanto, la fiabilidad se ha incrementado en $80,89 \%$.

\section{Tabla 8. Resultados para el indicador Fiabilidad}

Cantidad de respuesta sin errores a consultas de historiales médicos ambulatorios sin tecnología web por día

Cantidad de respuestas sin errores a consultas de historiales médicos ambulatorios con 1,63 tecnología web por día

Incremento de la Fiabilidad $8,53-1,63=6,9$

\% Fiabilidad $80,89 \%$

Para la variable dependiente en el grupo de control

\section{Para el indicador Eficiencia}

En esta investigación, la eficiencia fue medida en razón del índice Tiempo promedio en buscar un historial médico. Para garantizar que el tamaño de la muestra obtenido fuese representativo del trabajo observado, y que la magnitud del error y el riesgo admisible fuesen tolerables, se muestra la siguiente tabla descriptiva:

\begin{tabular}{lc}
\multicolumn{2}{c}{ Tabla 9} \\
\hline Población & finita \\
\hline Nivel de confianza & $95 \%$ \\
\hline Error estándar & $5 \%$ \\
\hline Nro. de observaciones & 48 \\
\hline
\end{tabular}

Fórmula aplicada:

$$
N=\left[\frac{40(48)}{704} \sqrt{\frac{10562-\frac{(704)^{2}}{48}}{48-1}}\right]^{2}
$$

Podemos observar que el tamaño de muestra representativa que se obtiene es de 38. En nuestro caso, se han recolectado 48 observaciones, lo que quiere decir que la cantidad observada es completamente representativa con respecto al resultado de la fórmula (ver anexo 7).

\section{Rango de clases y frecuencias}

Se formula la siguiente pregunta:

¿Cuál es el tiempo promedio en buscar un historial médico?

El tiempo promedio en buscar un historial médico desde el momento en que el paciente llega a la sala de espera hasta la atención propiamente dicha es aproximadamente de 15 minutos. Este tiempo muchas veces se prolonga hasta que llegue el encargado de transporte del historial médico.

La tabla 10 nos muestra las frecuencias del tiempo para atender a un paciente (grupo de control).

\section{Tabla 10. Tabla de frecuencias del tiempo} promedio en buscar un historial médico

\begin{tabular}{|rc|c|r|r}
\multicolumn{2}{|c|}{ Intervalo } & Frecuencia & $\begin{array}{r}\text { Punto } \\
\text { Medio }\end{array}$ & \multicolumn{1}{c|}{$\mathrm{Fr}^{*} \mathrm{X}$} \\
\hline 11,00 & 12,57 & 8 & 11,786 & 94,286 \\
\hline 12,57 & 14,14 & 13 & 13,357 & 173,643 \\
\hline 14,14 & 15,71 & 13 & 14,929 & 194,071 \\
\hline 15,71 & 17,29 & 8 & 16,500 & 132,000 \\
\hline 17,29 & 18,86 & 4 & 18,071 & 72,286 \\
\hline 18,86 & 20,43 & 1 & 19,643 & 19,643 \\
\hline 20,43 & 22,00 & 1 & 21,214 & 21,214 \\
\hline \multicolumn{2}{c}{ TOTAL } & 48 & TOTAL & $7 \mathbf{7 0 7 , 1 4 3}$ \\
\hline
\end{tabular}

El promedio es de 707,143 / $48=14,73$ minutos por búsqueda, equivalente a 15 minutos. 


\section{Donde:}

- El rango del intervalo se determina con la diferencia entre el valor máximo y el valor mínimo del número total de muestras observadas.

- El número de intervalos se halla empleando la regla de Estruges, en la que el número de intervalos es igual a: $1+3,3 \log (n)$, donde " $n$ " es el número de observaciones.

- La frecuencia se halló observando las muestras recopiladas y agrupándolas de acuerdo a los intervalos definidos.

- El punto medio es el resultado de la semisuma del valor mínimo y máximo de cada intervalo.

- La expresión $\left(\mathrm{Fr}^{*} \mathrm{X}\right)$ es el resultado de multiplicar la frecuencia (Fx) por el punto medio. Este factor nos permite calcular, conjuntamente con el tamaño de la muestra, la medía o promedio de las observaciones.

En la tabla 10 se han elegido 7 clases con un intervalo de 1,57 unidades, observándose que la clase de mayor frecuencia se encuentra ubicada en el intervalo 12,57 - 14,14 minutos.

\section{Estadistica descriptiva}

La tabla 11 nos ofrece, entre otra información, la frecuencia del tiempo promedio para buscar un historial médico (grupo de control).

El tiempo promedio en buscar un historial médico es de 28,735 minutos, y la mediana es de 15 minutos. Esta representa el número que se encuentra en medio de toda la muestra observada.

Además, se noto que el tiempo en promedio en buscar un historial médico con mayor frecuencia (moda) es de 15 minutos. Asimismo,
Tabla 11. Tabla de tiempo promedio en buscar un historial médico

\section{ESTADÍSTICA DESCRIPTIVA}

Media

28,735

Error típico

0,233

Mediana

15,000

Moda

15,000

Desviación estándar

2,244

Varianza de la muestra

5,035

Curtosis

1,047

Coeficiente de asimetría

0,873

Rango

11,000

Mínimo

11,000

Máximo

22,000

Suma

704,000

Cuenta

48,000

Mayor (1)

22,000

Menor(1)

11,000

Nivel de confianza

$(95,0 \%)$

el grado de dispersión (desviación estándar) que se obtuvo respecto de la medía fue 2,24, es decir, el tiempo de buscar un historial médico fluctúa entre 26,491 minutos y 30,979 minutos. La curtosis de 1,047 indica que la curva tiende a ser más aplanada con respecto a la curva normal.

A continuación se hace uso de la Prueba de Normalidad de Kolmogorov Smirnov para comprobar si se sigue o no una distribución normal. De acuerdo con la prueba mencionada, si la probabilidad del estadístico de contraste es mayor que 0,05 , se dice que la variable sí sigue una distribución normal, y si, por el contrario, la probabilidad es menor o igual que 0,05 no la sigue. A continuación vemos los resultados de la prueba realizada: 
Tabla 12. Prueba de Kolmogorov- Smirnov para el índice del tiempo promedio en buscar un historial médico

\begin{tabular}{lcr} 
& & MINUTOS \\
\hline $\mathrm{N}$ & & 48 \\
Parámetros normales $^{\text {a..b }}$ & Media & 14,6667 \\
& Desviación típica & 2,24398 \\
\hline $\begin{array}{l}\text { Diferencias más } \\
\text { extremas }\end{array}$ & Absoluta &, 149 \\
& Positiva &, 149 \\
Z de Kolmogorov-Simirnov &,- 097 \\
Sig. asintót. (bilateral) & Negativa & 1,034 \\
\hline
\end{tabular}

a. La distribución de contraste es la Normal.

b. Se han calculado a partir de los datos.

En esta tabla se observa una probabilidad de 0,235 , valor mayor a 0,05 ; por tanto, se confirma que se sigue una distribución normal.

\section{Para la variable dependiente en el grupo experimental}

\section{Para el indicador Eficiencia}

En esta investigación, la eficiencia se mide en razón del índice Tiempo promedio en buscar un historial médico. Para garantizar que el tamaño de la muestra obtenido fuese representativo del trabajo observado, así como para que la magnitud del error y el riesgo admisible resulten tolerables, se muestra la siguiente tabla descriptiva:

\section{Tabla 13}

\section{POBLACIÓN}

FINITA

Nivel de confianza $95 \%$

Error estándar $5 \%$

Nro. de observaciones 48

Se halló el tamaño de la muestra con la fórmula que hemos visto en la preprueba.

$$
N=\left[\frac{40(48)}{136} \sqrt{\frac{418-\frac{(136)^{2}}{48}}{48-1}}\right]^{2}
$$

Como podemos observar, el tamaño de muestra que se obtiene es de 44 atenciones. En nuestro caso, se han recolectado 48 observaciones, lo que significa que la cantidad observada es representativa con respecto al resultado de la fórmula (ver anexo 8).

\section{Rango de clases y frecuencias}

Se formula la siguiente pregunta:

¿Cuál es el tiempo promedio en buscar un historial médico?

El tiempo promedio en buscar un historial médico desde el momento en que el paciente llega a la sala de espera hasta la atención propiamente dicha es aproximadamente 3 minutos. Este tiempo se ha reducido significativamente debido a la utilización de la tecnología de información, que permite la atención del paciente en el momento más oportuno, minimizando el tiempo en la espera de la búsqueda. 
La tabla 14 nos muestra la frecuencia del tiempo promedio en buscar un historial médico (grupo experimental).

Tabla 14. Tabla de frecuencias del tiempo promedio en buscar un historial médico

\begin{tabular}{rr|r|r|r}
\multicolumn{2}{c|}{ INTERVALO } & FRECUENCIA & PUNTO MEDIO & \multicolumn{1}{c|}{ FR * $\mathbf{X}$} \\
\hline 2,00 & 2,43 & 20 & 2,214 & 44,286 \\
2,43 & 2,86 & 0 & 2,643 & 0,000 \\
2,86 & 3,29 & 0 & 3,071 & 0,000 \\
3,29 & 3,71 & 17 & 3,500 & 59,500 \\
3,71 & 4,14 & 0 & 3,929 & 0,000 \\
4,14 & 4,57 & 10 & 4,357 & 43,571 \\
4,57 & 5,00 & 1 & 4,786 & 4,786 \\
\hline \multicolumn{2}{r}{ TOTAL } & 48 & TOTAL & $\mathbf{1 5 2 , 1 4 3}$ \\
\hline
\end{tabular}

El promedio es de $152,143 / 48=3,170$ minutos por atención, equivalente a 3 minutos.

En la tabla 14 se han elegido 7 clases con un intervalo de 0,43 unidades, observándose que la clase de mayor frecuencia se encuentra ubicada en el intervalo de $2-2,43$ minutos.

\section{Estadistica descriptiva}

La tabla 15 nos da, entre otra información, el tiempo promedio para buscar un historial médico (grupo experimental).

\section{Tabla 15. Tabla de tiempo promedio para buscar un historial médico}

\begin{tabular}{lr} 
ESTADÍSTICA DESCRIPTIVA & \\
Media & 5,551 \\
\hline Error típico & 0,111 \\
Mediana & 3,000 \\
Moda & 2,000 \\
Desviación estándar & 0,834 \\
Varianza de la muestra & 0,695 \\
Curtosis & $-0,688$ \\
Coeficiente de asimetría & 0,558 \\
Rango & 3,000 \\
Mínimo & 2,000 \\
Máximo & 5,000 \\
Suma & 136,000 \\
Cuenta & 48,000 \\
Mayor (1) & 5,000 \\
Menor(1) & 2,000 \\
Nivel de confianza & $(95,0 \%)$ \\
\hline
\end{tabular}

El tiempo promedio de atención a un paciente es de 5,551, lo que significa que la aplicación de la tecnología de información, con la estrategia de respuesta efectiva, le permite al hospital una mayor eficiencia al momento de solicitar el servicio de búsqueda, donde la demora se producía por el tiempo que se perdía en no encontrar el historial médico, entre otros factores. Ahora se está en la capacidad de atender a un mayor número de pacientes en un menor tiempo posible.

Además, se notó que el tiempo en promedio para buscar un historial médico con mayor frecuencia (moda) es de 2 minutos Asimismo, el grado de dispersión (desviación estándar) que se obtuvo respecto de la medía fue de 0,834 , valor que resulta menor al hallado en la preprueba, lo que nos permite afirmar que los tiempos de búsqueda del historial médico tienen un menor grado de dispersión con respecto a los tiempos anteriores. Así pues, existiendo menos variabilidad en los datos, se puede deducir que el tiempo para atender a un paciente fluctúa entre 4,606 minutos y 6,274 minutos respectivamente. La curtosis de $-6,88$ nos indica que la curva es más aplanada con respecto a la preprueba, es decir, la nueva curva tiende a parecerse más a una curva normal.

A continuación se hace uso de la Prueba de Normalidad de Kolmogorov Smirnov para 
comprobar si se sigue o no una distribución normal. De acuerdo con la prueba mencionada, si la probabilidad del estadístico de contraste es mayor que 0,05 , se dice que la variable sí sigue una distribución normal, pero si, por el contrario, la probabilidad es menor o igual que 0,05 no la sigue. A continuación tenemos los resultados de la prueba realizada.

\section{Tabla 16. Prueba de Kolmogorov - Smirnov para el índice tiempo de atender a un paciente}

\begin{tabular}{lrr} 
& & MINUTOS \\
N & & 48 \\
Parámetros normales ${ }^{\text {a..b }}$ & Media & 2,8333 \\
& Desviación típica &, 83369 \\
Diferencias más & Absoluta &, 258 \\
extremas & Positiva &, 258 \\
& Negativa &,- 163 \\
Z de Kolmogorov-Simirnov & 1,787 \\
Sig. asintót. (bilateral) &, 003 \\
\hline
\end{tabular}

a. La distribución de contraste es la Normal.

b. Se han calculado a partir de los datos.

En la última línea de la tabla 16 se observa una probabilidad de 0,003 , valor menor a 0,05 ; por tanto, se confirma que no se sigue una distribución normal.

\section{Contrastación de hipótesis para el indicador de eficiencia}

Índice: Tiempo promedio en buscar un historial médico

Tomando en cuenta las estadísticas descriptivas para ambos grupos, tenemos:

\section{Tabla 17. Estadísticas. Tiempo promedio en buscar un historial médico}

\begin{tabular}{l|r|c}
\multicolumn{1}{c|}{ EFICIENCIA } & \multicolumn{2}{c}{ GRUPO } \\
\hline \multicolumn{1}{c}{ Datos estadísticos } & Control & Experimental \\
\hline Media & 28,735 & 5,551 \\
Desviación estándar & 2,244 & 0,834 \\
N $^{\circ}$ de observaciones & 48,000 & 48,000 \\
Grados de libertad & & 94 \\
\hline
\end{tabular}

Considerando que la muestra del grupo experimental sigue una distribución normal, se aplica la fórmula $t$-Student a los datos mostrados en la tabla 17, y se obtiene el siguiente resultado:

Tabla 18. Withney y prueba z. Tiempo promedio en buscar un historial médico

Ranks

\begin{tabular}{cl|c|c|c} 
& VAR00010 & N & MEAN RANK & SUM OF RANKS \\
VAR00009 & Grupo de control & 48 & 72,50 & 3480,00 \\
& Grupo experimental & 48 & 24,50 & 1176,00 \\
Total & 96 & & \\
\hline
\end{tabular}

\begin{tabular}{l|r}
\hline Test Statistics $^{3}$ & \\
& VAR00009 \\
Mann-Whitney U &, 000 \\
Wilcoxon W & 1176,000 \\
Z & $-8,523$ \\
Asymp. Sig. (2-tailed) &, 000 \\
\hline
\end{tabular}




\section{Interpretación}

Como se puede observar en la tabla 18 , el resultado de $\mathrm{z}$ es $-8,523$, y el de $\mathrm{p} \leq 0,000$ resulta significativo $(<0,05)$, lo que significa que se acepta la hipótesis de investigación para este índice, rechazándose, en consecuencia, la hipótesis nula. Esta aseveración queda expresada en los siguientes términos: La utilización de la tecnología web como soporte al proceso de seguimiento del historial médico ambulatorio de pacientes influye positivamente en la gestión administrativa del Hospital Central de la PNP de Lima Metropolitana.

\section{CONCLUSIONES Y RECOMENDACIONES}

A continuación se presenta una serie de planteamientos generales a manera de conclusiones y recomendaciones obtenidas en el desarrollo de la presente investigación.

\section{Conclusiones}

1. El tiempo promedio en buscar un historial médico sin la herramienta fue de 28,735 minutos. Después de la implementación de la herramienta, el tiempo fue de 5,551 minutos. Esto evidencia una reducción significativa de 23,184 minutos, lo que redunda en la satisfacción del paciente y de los médicos, además de permitir contar con información confiable y en tiempo real del paciente.

2. La hipótesis está contrastada porque la tecnología web como soporte al proceso de seguimiento del historial médico ambulatorio de pacientes influye positivamente en la gestión administrativa en el Hospital Central de la PNP de Lima Metropolitana.

\section{Recomendaciones}

1. Se debe contar permanentemente con el apoyo de los directores del Hospital de la PNP, así como también con la colaboración de las personas involucradas en el proceso de seguimiento del historial médico ambulatorio de pacientes, a fin de coadyuvar a un continuo mejoramiento del sistema.

2. Con respecto a la seguridad de la información, se debe tener en cuenta las normas para definir privilegios y permisos de usuarios, además de hacer backup periódicos de la información con la finalidad de salvaguardar su integridad.

3. Se debe capacitar en forma permanente al personal involucrado con el nuevo sistema, a fin de garantizar la eficiencia en su trabajo.

\section{REFERENCIAS BIBLIOGRÁFICAS}

1. Sánchez Carlessi, Hugo. Metodología y diseños en la investigación cientifica. Perú, Editorial Mantaro, 1999, 174 pp.

2. Hernández Sampieri, Roberto. Metodología de la investigación. 2da edición. México,1998

3. Nancy Victorio Gamarra. Tecnología intranet en la gestión del proceso de matriculas de la Universidad Alas Peruanas. Lima, Universidad Alas Peruana, 2003.

4. Saís Flores Hernández. Sistemas de gestión de documentos en la web. México, Instituto Politécnico Nacional, 2004.

5. Koontz Harold y Weihrich. Administración, una perspectiva global. Undécima edición. Heinz, McGraw Hill Interamericana Editores 1998, pp. 52-53.

6. Luis Fernández Suárez. Tecnología intranet en lagestión administrativa del Hospital Nacional Cayetano Heredia. Lima, Universidad Federico Villarreal, 2004. 


\section{Cuestionario}

Fecha $I$

Encuesta: Hospital PNP.

Provincia:

Area / Consultorio:

Nombre del entrevistado:

INTRODUCCION: La siguiente encuesta tiene como objetivo levantar información relevante de los pacientes del hospital de la PNP, ubicado en la av. Brasil cdra. 26 del distrito de Jesús Maria, con respecto a los tiempos en que se realiza el proceso de seguimiento del historial medico ambulatorio.

A.1. ¿Alguna vez, ha tenido que buscar personalmente su historial médico, al almacén de historiales médicos?
1. SI
2. NO
3. NS/NR

4. Otra

A.2. Alguna vez, se demoraron en atenderla/o porque no habia llegado su historial médico al consultorio?
1. SI
2. NO
3. NS/NR
4. Otra

A3. ¿Cuántas hojas tiene su historial médico?

1. 1 hoja 2. 2 hojas 3. Más de 3 hojas

A4. ¿Cuánto tiempo se demora desde que llega hasta que es atendido por el médico?
1. De $10-15$ minutos
2. De $15-20$ minutos
3. De 20- 25 minutos
4. Otra

A5. ¿Alguna vez, no se ha encontrado su historial mếdico en el hospital?
1. SI
2. NO
3. NSNR
4. Otra

A6 ¿Le gustaria que su información clínica se encuentre en una computadora?
1. Mucho
2. Bastante
3. Algo
4. Poco
5. Nada 
ANEXO 2

\section{GUIA DE ENTREVISTA}

Fecha:

Nombre del Participante:

Area de trabajo:

\section{LEVANTAMIENTO DE INFORMACION.}

INTRODUCCION: La siguiente guia de entrevista tiene como objetivo levantar información relevante de las personas involucradas en el proceso en estudio, "El proceso de seguimiento del historial médico ambulatorio".

- ¿Con que nombre se le conoce al documento en donde se registra la historia del paciente en el hospital?

- ¿Cómo un paciente registra un historial médico?

- ¿Diferencia entre un proceso ambulatorio y un proceso de emergencia?

- ¿En quẻ momento se actualiza las historias medicas de los pacientes?

- ¿Cuál es el flujo de la información después del registro del historial médico?

- ¿Cuentan con un sistema donde registren los historiales médicos?

- ¿Qué pasa si se pierde los documentos físicos de los historiales médicos?

- ¿Cómo están almacenados los historiales médicos?

OBSERVACIONES: 
ANEXO 3

CANTIDAD DE RESPUESTAS SIN ERRORES A CONSULTAS DE HISTORIALES MÉDICOS AMBULATORIOS SIN TECNOLOGÍA WEB POR DÍA

\begin{tabular}{|c|c|c|c|}
\hline OBS. & DÍAS & No DE RESPUESTAS SIN ERRORES & TOTAL^2 $^{\wedge}$ \\
\hline 1 & Día 1 & 10 & 100 \\
\hline 2 & Día 2 & 9 & 81 \\
\hline 3 & Día 3 & 7 & 49 \\
\hline 4 & Día 4 & 9 & 81 \\
\hline 5 & Día 5 & 9 & 81 \\
\hline 6 & Día 6 & 9 & 81 \\
\hline 7 & Día 7 & 7 & 49 \\
\hline 8 & Día 8 & 8 & 64 \\
\hline 9 & Día 9 & 10 & 100 \\
\hline 10 & Día 10 & 8 & 64 \\
\hline 11 & Día 11 & 9 & 81 \\
\hline 12 & Día 12 & 9 & 81 \\
\hline 13 & Día 13 & 7 & 49 \\
\hline 14 & Día 14 & 9 & 81 \\
\hline 15 & Día 15 & 9 & 81 \\
\hline 16 & Día 16 & 7 & 49 \\
\hline 17 & Día 17 & 7 & 49 \\
\hline 18 & Día 18 & 7 & 49 \\
\hline 19 & Día 19 & 8 & 64 \\
\hline 20 & Día 20 & 8 & 64 \\
\hline 21 & Día 21 & 9 & 81 \\
\hline 22 & Día 22 & 10 & 100 \\
\hline 23 & Día 23 & 8 & 64 \\
\hline 24 & Día 24 & 10 & 100 \\
\hline 25 & Día 25 & 9 & 81 \\
\hline 26 & Día 26 & 7 & 49 \\
\hline 27 & Día 27 & 8 & 64 \\
\hline 28 & Día 28 & 10 & 100 \\
\hline 29 & Día 29 & 10 & 100 \\
\hline \multirow[t]{2}{*}{30} & Día 30 & 9 & 81 \\
\hline & & 256 & 2218 \\
\hline
\end{tabular}

Fuente: elaboración propia 
ANEXO 4

\section{CANTIDAD DE RESPUESTAS SIN ERRORES A CONSULTAS DE HISTORIALES MÉDICOS AMBULATORIOS CON TECNOLOGÍA WEB POR DÍA}

\begin{tabular}{|c|c|c|c|}
\hline OBS. & DÍAS & No DE RESPUESTAS SIN ERRORES & TOTAL $^{\wedge} 2$ \\
\hline 1 & Día 1 & 2 & 4 \\
\hline 2 & Día 2 & 2 & 4 \\
\hline 3 & Día 3 & 2 & 4 \\
\hline 4 & Día 4 & 2 & 4 \\
\hline 5 & Día 5 & 2 & 4 \\
\hline 6 & Día 6 & 2 & 4 \\
\hline 7 & Día 7 & 2 & 4 \\
\hline 8 & Día 8 & 1 & 1 \\
\hline 9 & Día 9 & 1 & 1 \\
\hline 10 & Día 10 & 2 & 4 \\
\hline 11 & Día 11 & 1 & 1 \\
\hline 12 & Día 12 & 2 & 4 \\
\hline 13 & Día 13 & 2 & 4 \\
\hline 14 & Día 14 & 1 & 1 \\
\hline 15 & Día 15 & 1 & 1 \\
\hline 16 & Día 16 & 1 & 1 \\
\hline 17 & Día 17 & 2 & 4 \\
\hline 18 & Día 18 & 2 & 4 \\
\hline 19 & Día 19 & 1 & 1 \\
\hline 20 & Día 20 & 1 & 1 \\
\hline 21 & Día 21 & 2 & 4 \\
\hline 22 & Día 22 & 1 & 1 \\
\hline 23 & Día 23 & 2 & 4 \\
\hline 24 & Día 24 & 2 & 4 \\
\hline 25 & Día 25 & 2 & 4 \\
\hline 26 & Día 26 & 2 & 4 \\
\hline 27 & Día 27 & 1 & 1 \\
\hline 28 & Día 28 & 1 & 1 \\
\hline 29 & Día 29 & 2 & 4 \\
\hline \multirow[t]{2}{*}{30} & Día 30 & 2 & 4 \\
\hline & & 49 & 87 \\
\hline
\end{tabular}

Fuente: elaboración propia 
ANEXO 5

CANTIDAD DE HISTORIALES MÉDICOS AMBULATORIOS CON INFORMACIÓN ACTUALIZADA SIN ERRORES SIN TECNOLOGÍA WEB AL DÍA

\begin{tabular}{|c|c|c|c|}
\hline OBS. & DÍAS & $\begin{array}{l}\text { INFORMACIÓN ACTUALIZADA } \\
\text { CORRECTAMENTE }\end{array}$ & TOTAL $^{\wedge} 2$ \\
\hline 1 & Día 1 & 10 & 100 \\
\hline 2 & Día 2 & 12 & 144 \\
\hline 3 & Día 3 & 15 & 225 \\
\hline 4 & Día 4 & 12 & 144 \\
\hline 5 & Día 5 & 11 & 121 \\
\hline 6 & Día 6 & 13 & 169 \\
\hline 7 & Día 7 & 15 & 225 \\
\hline 8 & Día 8 & 10 & 100 \\
\hline 9 & Día 9 & 15 & 225 \\
\hline 10 & Día 10 & 12 & 144 \\
\hline 11 & Día 11 & 12 & 144 \\
\hline 12 & Día 12 & 11 & 121 \\
\hline 13 & Día 13 & 13 & 169 \\
\hline 14 & Día 14 & 14 & 196 \\
\hline 15 & Día 15 & 12 & 144 \\
\hline 16 & Día 16 & 9 & 81 \\
\hline 17 & Día 17 & 12 & 144 \\
\hline 18 & Día 18 & 14 & 196 \\
\hline 19 & Día 19 & 16 & 256 \\
\hline 20 & Día 20 & 8 & 64 \\
\hline 21 & Día 21 & 9 & 81 \\
\hline 22 & Día 22 & 11 & 121 \\
\hline 23 & Día 23 & 14 & 196 \\
\hline 24 & Día 24 & 10 & 100 \\
\hline 25 & Día 25 & 12 & 144 \\
\hline 26 & Día 26 & 11 & 121 \\
\hline 27 & Día 27 & 13 & 169 \\
\hline 28 & Día 28 & 14 & 196 \\
\hline 29 & Día 29 & 12 & 144 \\
\hline \multirow[t]{2}{*}{30} & Día 30 & 9 & 81 \\
\hline & & 361 & 4465 \\
\hline
\end{tabular}

Fuente: elaboración propia 


\section{ANEXO 6}

\section{CANTIDAD DE HISTORIALES MÉDICOS AMBULATORIOS CON INFORMACIÓN ACTUALIZADA SIN ERRORES CON TECNOLOGÍA WEB AL DÍA}

\begin{tabular}{|c|c|c|c|}
\hline OBS. & DÍAS & $\begin{array}{l}\text { INFORMACIÓN ACTUALIZADA } \\
\text { CORRECTAMENTE }\end{array}$ & TOTAL $^{\wedge} 2$ \\
\hline 1 & Día 1 & 2 & 4 \\
\hline 2 & Día 2 & 1 & 1 \\
\hline 3 & Día 3 & 2 & 4 \\
\hline 4 & Día 4 & 2 & 4 \\
\hline 5 & Día 5 & 1 & 1 \\
\hline 6 & Día 6 & 2 & 4 \\
\hline 7 & Día 7 & 1 & 1 \\
\hline 8 & Día 8 & 0 & 0 \\
\hline 9 & Día 9 & 1 & 1 \\
\hline 10 & Día 10 & 0 & 0 \\
\hline 11 & Día 11 & 1 & 1 \\
\hline 12 & Día 12 & 2 & 4 \\
\hline 13 & Día 13 & 0 & 0 \\
\hline 14 & Día 14 & 2 & 4 \\
\hline 15 & Día 15 & 1 & 1 \\
\hline 16 & Día 16 & 1 & 1 \\
\hline 17 & Día 17 & 2 & 4 \\
\hline 18 & Día 18 & 0 & 0 \\
\hline 19 & Día 19 & 0 & 0 \\
\hline 20 & Día 20 & 0 & 0 \\
\hline 21 & Día 21 & 1 & 1 \\
\hline 22 & Día 22 & 1 & 1 \\
\hline 23 & Día 23 & 1 & 1 \\
\hline 24 & Día 24 & 2 & 4 \\
\hline 25 & Día 25 & 2 & 4 \\
\hline 26 & Día 26 & 2 & 4 \\
\hline 27 & Día 27 & 2 & 4 \\
\hline 28 & Día 28 & 1 & 1 \\
\hline 29 & Día 29 & 0 & 0 \\
\hline \multirow[t]{2}{*}{30} & Día 30 & 2 & 4 \\
\hline & & 35 & 59 \\
\hline
\end{tabular}

Fuente: elaboración propia 


\section{ANEXO 7}

\section{TIEMPO PROMEDIO EN BUSCAR UN HISTORIAL MÉDICO EN MINUTOS (EFICIENCIA - CONTROL)}

\begin{tabular}{|c|c|c|}
\hline No OBS. & TOTAL MINUTOS & $(\text { MINUTOS })^{\wedge} 2$ \\
\hline 1 & 12 & 144,00 \\
\hline 2 & 15 & 225,00 \\
\hline 3 & 13 & 169,00 \\
\hline 4 & 15 & 225,00 \\
\hline 5 & 13 & 169,00 \\
\hline 6 & 15 & 225,00 \\
\hline 7 & 12 & 144,00 \\
\hline 8 & 19 & 361,00 \\
\hline 9 & 13 & 169,00 \\
\hline 10 & 13 & 169,00 \\
\hline 11 & 16 & 256,00 \\
\hline 12 & 12 & 144,00 \\
\hline 13 & 18 & 324,00 \\
\hline 14 & 22 & 484,00 \\
\hline 15 & 14 & 196,00 \\
\hline 16 & 18 & 324,00 \\
\hline 17 & 15 & 225,00 \\
\hline 18 & 13 & 169,00 \\
\hline 19 & 12 & 144,00 \\
\hline 20 & 14 & 196,00 \\
\hline 21 & 14 & 196,00 \\
\hline 22 & 15 & 225,00 \\
\hline 23 & 16 & 256,00 \\
\hline 24 & 14 & 196,00 \\
\hline 25 & 18 & 324,00 \\
\hline 26 & 17 & 289,00 \\
\hline 27 & 13 & 169,00 \\
\hline 28 & 12 & 144,00 \\
\hline 29 & 17 & 289,00 \\
\hline 30 & 16 & 256,00 \\
\hline 31 & 15 & 225,00 \\
\hline 32 & 18 & 324,00 \\
\hline 33 & 13 & 169,00 \\
\hline 34 & 13 & 169,00 \\
\hline 35 & 16 & 256,00 \\
\hline 36 & 13 & 169,00 \\
\hline 37 & 14 & 196,00 \\
\hline 38 & 15 & 225,00 \\
\hline 39 & 12 & 144,00 \\
\hline 40 & 15 & 225,00 \\
\hline 41 & 13 & 169,00 \\
\hline 42 & 15 & 225,00 \\
\hline 43 & 15 & 225,00 \\
\hline 44 & 16 & 256,00 \\
\hline 45 & 15 & 225,00 \\
\hline 46 & 12 & 144,00 \\
\hline 47 & 11 & 121,00 \\
\hline \multirow[t]{2}{*}{48} & 17 & 289,00 \\
\hline & 704,00 & 10562,00 \\
\hline
\end{tabular}

Estadística descriptiva

Media

Error típico

28,735

Mediana

0,233

Moda

15,000

Desviación estándar

15,000

Varianza de la muestr

2,244

Curtosis

5,035

Coeficiente de asimetría

1,047

Rango

0,873

Mínimo

11,000

Máximo

Suma

Cuenta

11,000

22,000

704,000

Mayor (1)

48,000

Menor(1)

22,000

Nivel de confianza

11,000

$\mathbf{N}=\quad 37,55$

$\mathrm{n}=$

$\mathrm{Sx}=$

$\mathrm{Sx}^{2}=$

Fuente: elaboración propia 


\section{ANEXO 8}

\section{MUESTREO GRUPO EXPERIMENTAL. INDICADOR DE EFICIENCIA TIEMPO PROMEDIO EN BUSCAR UN HISTORIAL MÉDICO EN MINUTOS (EFICIENCIA - GRUPO EXPERIMENTAL)}

\begin{tabular}{|c|c|c|}
\hline No OBS. & TOTAL MINUTOS & $(\text { MINUTOS })^{\wedge} 2$ \\
\hline 1 & 2,00 & 4,00 \\
\hline 2 & 3,00 & 9,00 \\
\hline 3 & 2,00 & 4,00 \\
\hline 4 & 3,00 & 9,00 \\
\hline 5 & 3,00 & 9,00 \\
\hline 6 & 3,00 & 9,00 \\
\hline 7 & 2,00 & 4,00 \\
\hline 8 & 2,00 & 4,00 \\
\hline 9 & 2,00 & 4,00 \\
\hline 10 & 3,00 & 9,00 \\
\hline 11 & 3,00 & 9,00 \\
\hline 12 & 4,00 & 16,00 \\
\hline 13 & 5,00 & 25,00 \\
\hline 14 & 4,00 & 16,00 \\
\hline 15 & 2,00 & 4,00 \\
\hline 16 & 3,00 & 900 \\
\hline 17 & 4,00 & 16,00 \\
\hline 18 & 2,00 & 4,00 \\
\hline 19 & 2,00 & 4,00 \\
\hline 20 & 2,00 & 4,00 \\
\hline 21 & 3,00 & 9,00 \\
\hline 22 & 3,00 & 9,00 \\
\hline 23 & 4,00 & 16,00 \\
\hline 24 & 4,00 & 16,00 \\
\hline 25 & 4,00 & 16,00 \\
\hline 26 & 3,00 & 9.00 \\
\hline 27 & 3,00 & 9,00 \\
\hline 28 & 3,00 & 9,00 \\
\hline 29 & 2,00 & 4,00 \\
\hline 30 & 2,00 & 4,00 \\
\hline 31 & 2,00 & 4,00 \\
\hline 32 & 3,00 & 9,00 \\
\hline 33 & 2,00 & 4,00 \\
\hline 34 & 2,00 & 4,00 \\
\hline 35 & 2,00 & 4,00 \\
\hline 36 & 2,00 & 4,00 \\
\hline 37 & 2,00 & 4,00 \\
\hline 38 & 2.00 & 4,00 \\
\hline 39 & 3.00 & 9,00 \\
\hline 40 & 4.00 & 16,00 \\
\hline 41 & 4.00 & 16,00 \\
\hline 42 & 4,00 & 16,00 \\
\hline 43 & 4,00 & 16,00 \\
\hline 44 & 3,00 & 9,00 \\
\hline 45 & 3,00 & 9,00 \\
\hline 46 & 3,00 & 9,00 \\
\hline 47 & 2,00 & 4,00 \\
\hline \multirow[t]{2}{*}{48} & 2,00 & 4,00 \\
\hline & 136,00 & 418,00 \\
\hline
\end{tabular}

\begin{tabular}{lr} 
Estadística descriptiva & \\
Media & 5,551 \\
Error típico & 0,111 \\
Mediana & 3,000 \\
Moda & 2,000 \\
Desviación estándar & 0,834 \\
Varianza de la muestra & 0,695 \\
Curtosis & $-0,688$ \\
Coeficiente de asimetría & 0,558 \\
Rango & 3,000 \\
Mínimo & 2,000 \\
Máximo & 5,000 \\
Suma & 136,000 \\
Cuenta & 48,000 \\
Mayor $(1)$ & 5,000 \\
Menor $(1)$ & 2,000 \\
Nivel de confianza & $95,0 \%)$ \\
\hline & \\
$\mathrm{N}=$ & 138,53 \\
$\mathrm{n}=$ & 48 \\
$\mathrm{Sx}=$ & 136,00 \\
$\mathrm{Sx}{ }^{2}=$ & 418,00 \\
\hline
\end{tabular}

Fuente: elaboración propia 